\title{
A Secure and Intelligent Framework for Vehicle Health Monitoring Exploiting Big-Data Analytics
}

\author{
Md. Arafatur Rahman*, Senior Member, IEEE, Md. Abdur Rahim, Md. Mustafizur Rahman*, Nour Moustafa, \\ Imran Razzak, Tanvir Ahmad, and Mohammad N. Patwary, Senior Member, IEEE,
}

\begin{abstract}
The dependency on vehicles is increasing tremendously due to its excellent transport capacity, fast, efficient, flexible, pleasant journey, minimal physical effort, and substantial economic impact. As a result, the demand for smart and intelligent feature enhancement is growing and becoming a prime concern for maximum productivity based on the current perspective. In this case, the Internet of Everything (IoE) is an emerging concept that can play an essential role in the automotive industry by integrating the stakeholders, process, data, and things via networked connections. But the unavailability of intelligent features leads to negligence about proper maintenance of vehicle vulnerable parts, reckless driving and severe accident, lack of instructive driving, and improper decision, which incurred extra expenses for maintenance besides hindering national economic growth. For this, we proposed a conceptual framework for a central VHMS exploiting IoE-driven Multi-Layer Heterogeneous Networks (HetNet) and a machine learning technique to oversee individual vehicle health conditions, notify the respective ownerdriver real-timely and store the information for further necessary action. This article transparently portrayed an overview of central VHMS and proposed the taxonomy to achieve such an objective. Subsequently, we unveiled the framework for central VHMS, IoE-driven Multi-tire HetNet, with a secure and trustworthy data collection and analytics system. Finally, anticipating this proposition's outcome is immense in the automotive sector. It may motivate the researcher to develop a central intelligent and secure vehicular condition diagnostic system to move this sector towards Industry 4.0.
\end{abstract}

Index Terms-Central VHMS, IoE, HetNet, Machine Learning, VehiChain.

\section{INTRODUCTION}

$\mathbf{T}$ $\mathrm{HE}$ vehicle is an essential item in our daily lives due to its enormous beneficial aspects and makes our lives easier by solving many complex confronted tasks. So, it

Manuscript received April July 23, 2021; revised October 07, 2021; accepted December 14, 2021. The authors would like to thank University Malaysia Pahang for providing the laboratory facilities and financial support under the University FLAGSHIP Research Grants (Project number RDU192203), International Matching Grant (No. RDU192704), and Postgraduate Research Scheme Grant (No. PGRS200325)

M. A. Rahman and Mohammad N. Patwary are with School of Mathematics and Computer Science, University of Wolverhampton, Wulfruna St, Wolverhampton WV1 1LY, UK. (e-mail: arafatur.rahman@ieee.org, patwary@wlv.ac.uk).

M. A. Rahim, M. M. Rahman and Tanvir Ahmad are with the University Malaysia Pahang, 26300 Gambang, Kuantan, Pahang, Malaysia. (e-mail: mdabdurrahim.me2k7@gmail.com, mustafizur@ump.edu.my, tanvir_700@yahoo.com).

Nour Moustafa is with UNSW Canberra, Australia. (e-mail: nour.moustafa@unsw.edu.au).

Imran Razzak is with Deakin University, Australia. (e-mail: imran.razzak@deakin.edu.au).

*Corresponding authors is considered one of the revolutionary inventions of the modern age and the development of our present condition. Fundamentally these depend on vehicles' efficient usage and preventive measures [1]. While uttering about efficient vehicle usage, some terms come accordingly, such as efficient traffic management, ensuring road safety, proper city management, perfect vehicular condition observation, proper maintenance, and many other related services. In this case, a prominent invention is required that may perfectly fulfil these matters. Based on the current perspective, this appeals to researchers to design a monitoring system that rigorously studies a vehicle's health intelligently and informs respective stakeholders about the vehicle's structural conditions in real-time.

Currently, the most circulated term is intelligence, and it is a groundbreaking concept that every sector of modern technologies is adopting these techniques to improve the system's performance [2]. An intelligence system is a decisionaware technique that makes a system adapt to any unknown or unfamiliar situations. For diagnosing the fault of a machine [3] or operation of a hospital, or even managing a city [4], worldwide, the intelligence system proves its ability. The application of this technique to the vehicle and related fields is enormous. Design of self-contained autonomous vehicles [5], telematic feature contained vehicle [6], enhanced intelligent transport system [7], and so forth are the unreal things that come to reality by utilizing intelligence techniques. However, in the age of Industry 4.0, the world is submerged with data [8]. Such as the amount of data produced by an online social network like Facebook, Twitter, and others are mammoth. So, it is inevitable that data originated from wireless and other systems would be huge too. This massive amount of data can be termed as 'Big Data', as a repository where dimensions and essences of data are assorted from one another [9]. Due to various benefits, the big data for vehicle applications attract academicians, researchers, vehicle manufacturers, environmentalists, and many other agencies [10]. On the contrary, the security issue was not addressed accordingly while designing networking systems from the very beginning of its emergence [11]. The possibility of an intruder attack on any communication system has increased so viciously; as a result, people deny receiving any services from the system without trustworthy security measures. The vehicular communication infrastructure is highly sensitive, where researchers conducted abundance praiseworthy research to ensure its security features in different application cases and lots of research is ongoing. It can be presumed that intelligence and security are the two most asking requirements of any 
system. In short, both are prerequisites to design a system that has multiple applicabilities and access to other networks and systems [12].

However, the vehicle has lots of essential components, and those are keeping a role a little or more to make it operational over the road. Moreover, among these parts, some parts could be more essential components, and minor ignorance could lead to a devastating situation. The noticeable matter is that these items produced a massive quantity of data for every second. Recently some research has been conducted to observe these items, but these works are confined to simple and individual cases using contemporary sensor-actuator. So, Vehicle Health Monitoring System (VHMS) for a central entity besides individual circumstances could be considered a unique venture. The notable matter is the available recent studies are not concentrated on this concept. In [13], the researchers concentrated only on locating the vehicle and identifying the vehicle's fuel level. Again Shafi et al. [14] focused on few specific engine issues using IoT technology instead of considering the whole vehicle. However, these studies have several loopholes and are inconsistent with the concept of Industry 4.0. Also, in, [13], the GPS and GSM were used for communication purposes for the vehicle monitoring system, which makes the system incompetent compared to the current situation. Also, GSM technology treats this system as a secondary system that can not ensure the quality of service (QoS), and there is no option for improving the system's performance. Secondly, only fuel monitoring can not be considered as a parameter for ensuring a perfect monitoring system. Again, in some cases, researchers started to employ the most popular machine learning techniques like artificial intelligence (AI) for realtime remote monitoring [14], but the data were taken from OBD-II instead of contemporary sophisticated devices, which seems ineffectual with the current Industry 4.0 demand. Also, the authors of these works overlooked implementing these propositions for the central VHMS entity using multi-variant connectivity contained networks besides considering security issues. Overally, the previous research works employed data collection techniques, networking devices and monitoring approaches are obsolete and unsuitable for the current perspective. Keeping in mind these problems, an intelligent and secure vehicle health monitoring system is needed to be designed, which facilitates the advantage of a reliable and robust network system using Internet of Everything (IoE) technology.

So, this study proposed a central VHMS for real-time monitoring systems that monitor the vehicle's health status and simultaneously notified the stakeholders and store this information besides providing telematics services, storing driverowners data. It can be further exploited by stakeholders such as the Road Transport Department (RTD), vehicle manufacturers, owners, insurance authorities, government agencies, manufacturers, etc. In this case, the VHMS may facilitate the stakeholders by incorporating cloud-based services where the VHMS can be handled centrally. The network infrastructure formation could be governed by client-server techniques where the data can be propagated from vehicle to cloud through the fog network and vice versa by following Figure 11 However, this paper describes some conceptual issues of central VHMS elaborately with some advanced and sophisticated features, which may enlighten the reader to more insights into that particular issue. Virtually, this paper concentrates on the following matters:

1. Portrayed an overview of central VHMS including IoEdriven Multi-Tire HetNet with secured network system;

2. Proposed taxonomy towards the possibility of central VHMS through the extensive investigation on literature;

3. Presented a conceptual framework for central VHMS to motivate future researchers to enhance the automotive sector with advanced technology;

4. Discussed a set of open research issues to deliver novel ideas among researchers to enhance the central VHMS.

Moreover, the remaining portion of the paper is decorated with different topics. Section II proposes a taxonomy of this paper with descriptive features, and it's a related literature review. The uniqueness of this research is portrayed in Section III as the VHMS framework to a central extent. The future research scope and challenges are elucidated in Section IV to share the novel ideas with the respected researchers. Finally, Section $\mathrm{V}$ concludes the paper by disclosing the contribution of the article.

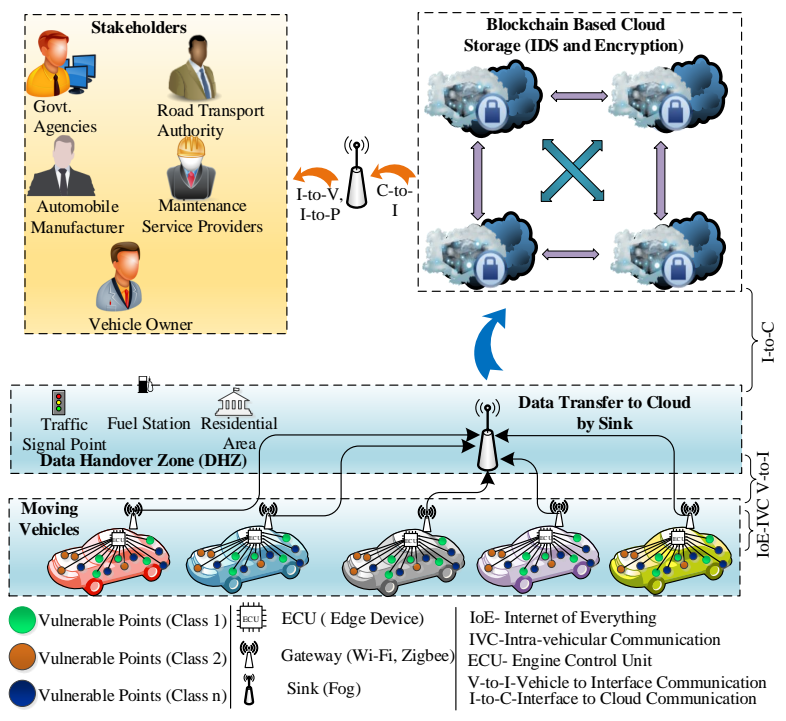

Fig. 1. Overview of Secure and Intelligent VHMS Framework by Big-Data Analytics.

\section{PRoposed TAXONOMY}

The application of central VHMS may effectively influence the path of development and make comfortable our everyday life. For this, a birds-eye view of the different concept's relation is presented through the taxonomy in Figure 2.

\section{A. Intelligence Concept for VHMS}

Intelligence is the virtue of any entity where it can predict the viable decision as outcomes depending on the gathered knowledge. In VHMS, this term is considered a promising and novel item to make decisions about any arisen matters smartly in the following cases. 


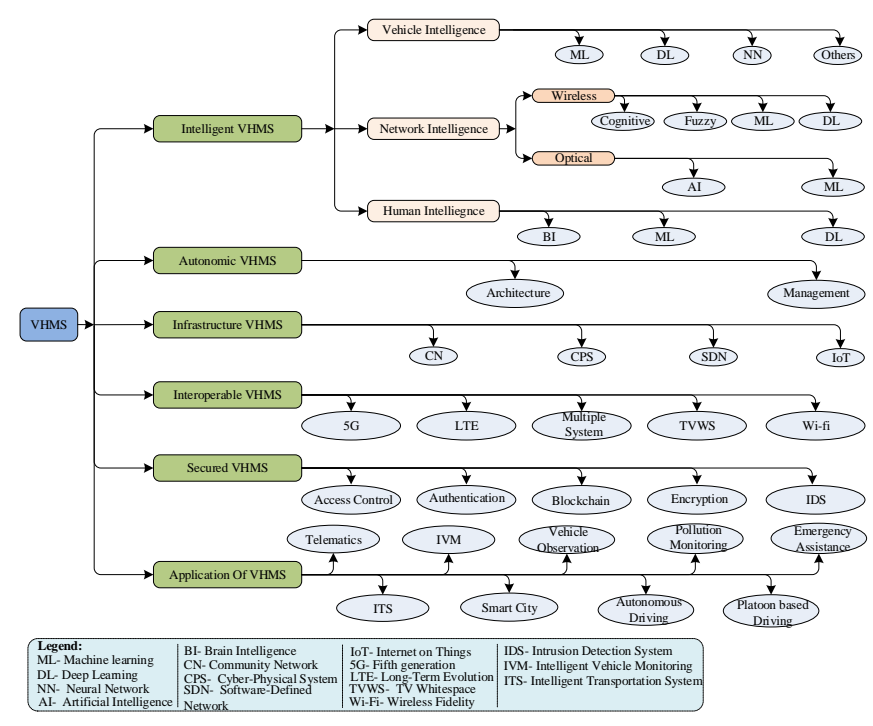

Fig. 2. Proposed Taxonomy for VHMS

1) Intelligence on Vehicular Communication: Communication technologies are the most significant component of sharing the data perfectly among vehicles for various purposes, where the following techniques could enhance the analytic activities to share the desired information intelligently.

a) ML-based Intelligent Vehicular System: ML-based intelligence is exploited in many aspects in vehicular networking, vehicular communication, and securing vehicular communication [15]. In [16], several techniques are proposed, such as Supervised Learning, Unsupervised Learning, Reinforcement Learning, Deep Learning (DL) as supreme ML tools to design a vehicular communication system. In this case, Multi-Layer Perception (MLP), Support Vector Machine (SVM), K-Nearest Neighbor (KNN) algorithms are utilized for a better decision [17]. Besides, a novel reinforcement learning-based algorithm, i.e., Deep Reinforcement Learning (DRL) based algorithm, Artificial Neural Network (ANN), and Hidden Markov Model (HMM) based time series ML algorithm also used for intelligent networking allocation by establishing Software Defined Network (SDN) incorporating with Mobile Edge Computing (MEC) and Network Function Virtualization (NFV). In this case, the developed system is worked as an integral part of network resource allocation, network traffic allocation, and network virtualization. Additionally, the Q learning-based algorithm, Bayes learning-based algorithm, and the K-means unsupervised algorithm are also used to cover many security features like intrusion detection, malicious attacks, and hybrid detection [18].

b) DL-based Intelligent Vehicular System: Though DL is considered a subset of ML, but it secures a distinctive position for its effective and extensive practices towards AI's journey. Such as MLP, Recurrent Neural Network (RNN), Convolutional Neural Network (CNN), Graph Convolutional Network, Autoencoders (AE), and so forth are efficient methods which recently have been incorporated in the intelligent transportation systems (ITS) for optimal outcomes [19].

c) Neural Network-based Intelligent Vehicular System:

Neural Network (NN) is another special type of machine learning technique. It is renowned for its predicting performance accuracy in many sectors, including in ITS. The notable matter is that NN sets its footprints in the field of automated driving, which is proposed in [19]. Also, NN-based CNN decorates ITS for traffic sign recognition, traffic light detection, vehicle classification, number plate/license recognition, etc., which are discussed eloquently in [20]. Additionally, based on the current perspective for lightweight and more effective modern techniques, the defogging algorithm-based image processing technique, image processing embedded machine vision method, high-resolution camera dependent vision sensing method [21] are proposed for designing an intelligent vehicle and ITS.

2) Intelligence on Network Technology: Different kinds of technologies are adopted based on the adjacent environment, requirements, cost, and other necessities to achieve the expected goal using various intelligence algorithms for optical networking and wireless networking, which are described as follows.

a) Intelligence on Optical Network: In this case, the Bayesian filtering with expectation maximization, genetic algorithms, and adaptive control approaches are used to characterize and operate the optical transmitters. On the other hand, ANN and DNN are applied for performance monitoring of optical networks, where Bayesian filtering with expectationmaximization is adopted for mitigations of nonlinearities. Again, for enhancing the Quality of Transmission of this networking system, the Case Base Reasoning (CBR) technique is performed outstandingly as a potential AI technique. Another notable matter is that the Deepwalk strategy is employed for optical network slicing and management purposes, and the Deep Neural Evolution Network (DNEN) to identify fault locations perfectly [22]. On the other hand, the Erbium-Doped Fiber Amplifier (EDFA) operation adopted ML techniques like supervised machine learning used in the optical network for efficient outcomes. Also, for calculating the Quality of Transmission of the optical network efficiently the DNN, and ANN-based ML are used profoundly [23].

b) Intelligence on Wireless Network: Due to the complexity and critical network consideration, the intelligence feature is most vastly used in a different instances of wireless networking. Such as, in the early stage of wireless intelligence, the cognition system was predominant solely, and it was massively used before the era of modern intelligent techniques. For example, Cognitive Radio (CR) is used mainly for spectrum sensing, besides various sensing purposes like energy estimation, matched filter detection, feature detection, etc. are used for this purpose [24], [25]. With changing time, the ML technique achieved popularity for managing wireless communication. For example, power control, spectrum management, cache management, backhaul management, and so forth activities are competently managed using various approaches of ML such as supervised learning, reinforcement learning, and transfer learning. Again for wireless networking, the base stations (BS) association, BS switching, routing, and clustering are adopted using different ML-based approaches. 
TABLE I

COMPARATIVE ANALYSIS OF INTELLIGENCE

\begin{tabular}{|c|c|c|c|c|c|c|c|c|c|c|c|c|c|}
\hline \multirow{3}{*}{$\begin{array}{l}\text { Different features exploited } \\
\text { by Intelligence }\end{array}$} & \multicolumn{4}{|c|}{ Intelligence in vehicle } & \multicolumn{6}{|c|}{ Intelligence in-network Technology } & \multicolumn{3}{|c|}{ Intelligence in vehicle } \\
\hline & & & & & & Wir & eless & & & tical & & & \\
\hline & ML & DL & NN & Others & Cognitive & Fuzzy & ML & DL & AI & ML & BI & DL & ML \\
\hline Spectrum Sensing & $\checkmark \quad 18$ & $\mathrm{X}$ & $\mathrm{X}$ & $\mathrm{X}$ & $\checkmark 24$ & $\mathrm{X}$ & $\checkmark \quad 29$ & $\mathrm{X}$ & $\mathrm{X}$ & $\mathrm{X}$ & $\mathrm{X}$ & $\mathrm{X}$ & $\mathrm{X}$ \\
\hline Channel Estimation & $\checkmark \overline{18}$ & $\checkmark \quad 30$ & $\mathrm{X}$ & $\mathrm{X}$ & $\mathrm{X}$ & $\mathrm{X}$ & $\mathrm{X}$ & $\checkmark 27$ & $\mathrm{X}$ & $\mathrm{X}$ & $\mathrm{X}$ & $\mathrm{X}$ & $\mathrm{X}$ \\
\hline $\begin{array}{l}\text { Radio Resource Allocation } \\
\& \text { Management }\end{array}$ & $\checkmark \overline{16}$ & $\checkmark[\overline{31}$ & $\mathrm{X}$ & $\mathrm{X}$ & $\checkmark \quad 25$ & $\mathrm{X}$ & $\checkmark \mid 32$ & $\checkmark \quad \overline{27}$ & $\mathrm{X}$ & $\mathrm{X}$ & $\mathrm{X}$ & $\mathrm{X}$ & $\mathrm{X}$ \\
\hline Network Traffic Control & $\checkmark 16$ & $\checkmark \quad 33$ & $\mathrm{X}$ & $\mathrm{X}$ & $\mathrm{X}$ & $\mathrm{X}$ & $\mathrm{X}$ & $\checkmark 27$ & $\mathrm{X}$ & $\checkmark \longdiv { 2 3 }$ & $\mathrm{X}$ & $\mathrm{X}$ & $\mathrm{X}$ \\
\hline Routing & $\mathrm{X} \| \overline{18}$ & $\checkmark[\overline{34}$ & $\mathrm{X}$ & $\mathrm{X}$ & $\mathrm{X}$ & X & $\checkmark 29$ & $\checkmark \quad \overline{27}$ & X & $\checkmark \longdiv { 2 3 }$ & X & $\mathrm{X}$ & X \\
\hline
\end{tabular}

Also, in some cases notably, unsupervised learning, supervised learning, and reinforcement learning approaches of ML are practiced significantly to ensure mobility management of wireless systems [26]. Besides the ML, in the physical layer of wireless communication system, several issues such as Interference Alignment (IA), jamming resistance, modulation classification, and physical coding are resolved using deep Q-learning, deep Q-learning based two-dimensional antijamming scheme, CNN based DL scheme, decoding algorithm and so forth of DL techniques. Some problems like spectrum allocation, traffic prediction, and link evaluation are also successfully resolved in the data link layer by Dbased advanced schemes such as hybrid DL, Deep Belief Network (DBN), and so forth. Besides these, in the routing layer, DL-based Q-learning and deep reinforcement learning have been used magnificently in many studies [27]. Also, in various applications of wireless communication systems, i.e., wireless virtual reality, co-existence of multiple radio access technology, mobile edge caching and computing, the NN based Echo State Network (ESN), CNN, Long Short Term Memories (LSTM), Feed Forward Network (FNN), and so forth have exploited as next-generation wireless application [28].

3) Intelligence-based Human Science: The intelligence concept used in different science and technology branches originated from biological science, especially medical science. So it is obvious to look for novel concepts in biological science.

a) Brain Intelligence (BI): Brain Intelligence (BI) can also be enunciated as Beyond AI. Generally, it is incorporated by AI and artificial life features with memory, where the artificial life-based network connects different NN. Though, the traditional AI approaches forget the previous method while learning the new one. But Continual Learning as BI keeps the previous one in the memory while learning the new approaches, such as Elastic Weight Consolidation (EWC) [35].

b) ML-DL Approaches and Human Science: ML techniques like the tree-based method, SVM, and others are widely used to treat cardio-related diseases. Additionally, in various clinical purposes like radiology, hematology, neurology, oncology, and cardiology used different ML-based approaches efficiently [36]. On the other hand, DL approaches are adopted for detecting and treatment purposes in various ophthalmology problems like diabetic retinopathy, age-related macular degeneration, and glaucoma. Additionally, DL-based CNN is widening the using scopes of intelligence for medical imaging and human emotion recognition (HERO) in the radiology field [37].
4) Comparative Analysis of Intelligence: So far, the intelligence concept discussed in various aspects of different applications makes anyone understand that this field is quite vast and versatile. Concentrating on the application of VHMS, the analytical discussion of intelligence is focused on the networking of the VHMS. From Table I, various networking component is considered, which are thriving factors to design a robust and efficient network. From the analysis, ML and DL become the two strong contenders for consideration. Surprisingly, NN lags behind the race. In this case, the true facts lie in the complexities of solving capabilities and functions of the employed algorithms. But the $\mathrm{NN}$ algorithms are designed to solve critical problems like behavior analysis and facial recognition, which require higher computation and resources. Cognitive intelligence and fuzzy intelligence are not capable of performing a task like ML and DL at all. However, the research to incorporate Brain Intelligence and other Human Science intelligence into physical technology is ongoing.

\section{B. Autonomic Concept for VHMS}

As a new concept, Autonomic Computing (AC) has been derived from the idea autonomous nervous system of the human body where the human body sense an abnormal situation, cope with it and resolve the situation by manipulating internal mechanism [38]. The $\mathrm{AC}$ has its potential towards the manageability of disaster. The Autonomic concept more novel than automatic, where no human or outside intervention is needed to handle the crucial situation for any system.

1) Architecture: A Generic Autonomic Network Architecture (GANA) model is proposed, where the GANA profile is created from the interaction between autonomic nodes, and these profiles are extracted by analyzing autonomic behavior. There are several Decision Elements (DE), which are constructed protocol, function, network, and node. Another GANA model inspired Autonomic Network Integrated Model and Architecture (ANIMA) is proposed, where autonomic networks have three layers. Each autonomic network is formed by autonomic service agents, autonomic network infrastructure, and basic operating system functions, which hold a globally unique domain certificate. However, an autonomic architecture-based database-centric architecture named Relational Model Multiagent system (RMAS) by ensuring selfhealing, self-configuration, and self-optimization, besides selfprotection features [39].

2) Management: Autonomic Computing makes itself worthy of managing many aspects like data handover, cloud 
computing, and QoS. In this case, the ML approaches in autonomous computing also make management intelligent [38], [40].

\section{Infrastructure Concept for VHMS}

Infrastructure holds a vital share for building a network, and the performance of a wireless network depends on the appropriate infrastructure design. In search of finding a suitable infrastructure, the following discussions might shed some light on this topic.

1) Community Network: Community Network $(\mathrm{CN})$ is constructed from the concept of open source and its uses. The idea came from the crowdsourced network, and it is devised to be open, free, and neutral. For building a $\mathrm{CN}$ infrastructure, it is necessary to define the technology and a network infrastructure layer. Guifi.net is an established example of $\mathrm{CN}$ infrastructure where people's Wi-Fi and fibre-optic plays as a last-mile deployment. Such as, in [41], CN-based network infrastructure is implemented to build connectivity in a rural environment. Besides that, Wireless $\mathrm{CN}$ is also proposed by a bottom-up approach for creating an excellent $\mathrm{CN}$ infrastructure.

2) Cyber-Physical System: Cyber-Physical System (CPS) amalgamates computational and physical components to form a process in the real world [42]. In a broad sense, in terms of Architecture, the user interacts with CPS through the Cyber part interface, and cyber coordinates and controls the physical part through a properly designed network. CPS is a modernday technology that takes the automation system a leap forward [43]. The capability to handle different physical systems makes it a worthy candidate to operate in the heterogeneous network. The whole system is classified into two domains; Cyber and Physical, where sensors and actuators work as components in the physical domain, and computation is done in the cyber domain, which is coordinated by communication between sensing and control [44]. In [45], a Structural Health Monitoring System (SHMS) in an aircraft is implemented where a piezoelectric transducer works in the physical domain, and Wireless Sensor Network (WSN) plays the main role in the cyber domain. Also, a MegaSense system for monitoring air quality was proposed by [46], where the MegaSense core plays as a cyber system and the air pollution monitoring system works as a physical platform. Besides these, a CPS system with the association of social networking is used to design an Internet of Vehicle (IoV) where data collection and networking layer are incorporated in the socio-physical layer and data processing, fusion, knowledge discovery layer assembled in the cyber layer [47]. Moreover, Cyber-physical scheduling for inter-vehicle communication, CPS-based autonomous valet parking, cooperative driving, and lane changing for connected vehicles are some recent advancements of CPS applications for the vehicles. Security measures for event detection also can be adopted in CPS [48].

3) Internet of Things: In remote VHMS infrastructure, the Internet of Things (IoT) is crucial for implementing as a constructive item to perform the information sharing operation real-timely [49]. It has three layers: application, perception, and network layer, which are the basic layers that create the general architecture of IoT [50]. Besides that, four more layers to equip IoT are more competent to accommodate diverse technologies, including the environment, communication, service, and management [51]. The prime objective of IoT is to interconnect various vehicles from networks and control a massive number of devices [52]. For designing the future internet of the vehicle [53], SDN-based IoT as a virtualization technique is proposed where a virtualization layer is incorporated with the data, control, and application layer. A novel Location-based Urban Vehicle (LUV) network is developed by keeping the vehicle's datalinks between places of residence and visiting places, where the conventional strategy is used to compute routes between destinations and starting places. Recently a seven-layer model architecture for IoT-based IoV has been proposed to enhance vehicular communication [54]. Besides these, sensing, networking, cloud computing, and application layers are considered for designing four-layer heterogeneous IoT as the Internet of Everything (IoE) to ensure the connectivity of all available nodes with the internet [55], [56].

4) Software-Define Network: Software-Defined Network (SDN) comes from an idea to separate the control logic of network infrastructure from the data plane. The data plane consists of devices and traffics that participate in forwarding the data. SDN logically divides the whole infrastructure into network application and control logic, where it plays as the decisional node. Actually, SDN virtualized the network function, for which it can be termed as Network Function Virtualization (NFV). SDN-based network infrastructure Ethanol [57] proposes enabling the access point to attain more control accuracy. In [58], SDN is proposed for IoV, where the SDN part function as a control plane for designing the infrastructure. In this case, different policies and algorithms are integrated into the control part to manage the overall infrastructure. Also, a distinctive network architecture based on SDN solely for the vehicular network is prototyped, where the SDN backbone layer works as an interconnection between vehicles and the internet [59]. Besides these, a multi-level SDN is formed by the Local SDN Controller and Central SDN Controller (CSDNC) for 5G VANET's to manage the system more efficiently and ensures its scalability [60].

5) Comparative Analysis of Infrastructure: Numerous factors can be evaluated while constructing a stalwart infrastructure for VHMS based on the above study on infrastructure. To be more precise, every trifling thing can be a factor in designing excellent VHMS infrastructure. However, the identified factors of different infrastructures are presented in Table II to ensure better VHMS infrastructure implementation in central entities.

A layered architecture is a prerequisite to materialize a robust infrastructure. But the $\mathrm{CN}$ has some lackings in structure and layered architecture because it originated from the concept of volunteering, which makes it evident that it cannot have a predefined architecture. Adopting different communication technologies is a prime necessity for any wireless network to the channel's random nature. Also, it is evident that the input and output of the layers have a substantial impact on the performance of infrastructure. A coherent input/output such as data rate and proper knowledge of channels is important for 
TABLE II

COMPARATIVE ANALYSIS OF INFRASTRUCTURE

\begin{tabular}{|c|c|c|c|c|}
\hline Features of Infrastructure & $\mathrm{CN}$ & SDN & CPS & IoT \\
\hline $\begin{array}{l}\text { Predefined Layered Ar- } \\
\text { chitecture }\end{array}$ & $\mathrm{X}$ & $\checkmark[\overline{59}]$ & $\checkmark[\overline{44]}$ & $\checkmark \mid 54$ \\
\hline $\begin{array}{l}\text { Deployed Various Com- } \\
\text { munication Technology }\end{array}$ & $\checkmark 61$ & $\checkmark 60$ & $\checkmark 62$ & $\checkmark 63$ \\
\hline $\begin{array}{l}\text { Interaction among Lay- } \\
\text { ers }\end{array}$ & $\checkmark 41$ & $\checkmark[57$ & $\checkmark 47$ & $\checkmark 64,65$ \\
\hline $\begin{array}{l}\text { Structured Input and } \\
\text { Output at every Laver }\end{array}$ & $\mathrm{X}$ & $\checkmark[58]$ & $\checkmark[46]$ & $\checkmark 66$ \\
\hline $\begin{array}{l}\text { Multiple } \\
\text { less/wired) }\end{array}$ & $\checkmark 41$ & $\checkmark[59]$ & $\checkmark$ 47] & $\checkmark 53$ \\
\hline $\begin{array}{l}\text { Communication Medium } \\
\text { Integration with other } \\
\text { System }\end{array}$ & $\mathrm{X}$ & $\checkmark[60]$ & (62] & $\checkmark 67$ \\
\hline Virtualization & $\mathrm{X}$ & $\checkmark[68$ & $\mathrm{X}$ & $\checkmark 55$ \\
\hline
\end{tabular}

infrastructure to function properly, often not present in $\mathrm{CN}$ for random architecture. Infrastructures adopt the idea of multiple connectivities with associated nodes to maintain backhaul or backbone network connectivity. Another mentionable matter is that the complexity of infrastructure is increasing daily due to the heterogeneous nature of devices and virtualization. In this case, IoE is the perfect option for developing a robust infrastructure.

\section{Security Approaches for VHMS}

Security measures must be robust and reliable since the users may gain access to several independent entities to be benefited from desired purposes [51]. Generally, it is ensured based on the triad concept known as CIA; Confidentiality, Integrity, and Authentication [2]. In central VHMS, for ensuring security, the following approaches have great possibilities.

1) Access Control Based Security: Security enhancement for WSN affirmed by resisting message reply attack, and Sybil attack using Practical Access Control Protocol (PACP) [69]. Besides, Authorizing Workflow Task Role Base Access Control (AW-TRBAC) is also proposed as the extension of RoleBased Access Control. It is formed by several components, considers granting user access policy through role change in a dynamic fashion [70]. For IoT security, a novel attributebased access control scheme for each device is provided in [71], where no device is allowed to access the system until it gets enough attributes. In this case, Blockchain technology is utilized to record the distribution of attributes among the attributes devices. Another novel Security and Privacy Policybased Access Control (SPBAC) is proposed for vehicular communication to overcome the segregation of duty problems so that Dynamic Segregation Duty (DSD) can be implemented perfectly [72].

2) Authentication Based Security: The authentication scheme for WSN based Anonymous Access Authentication (AAA-WSN) is designed on the concept of a single key for every authentication session [73]. For IoV based authentication, an improvement of Authentication based on Smart Card (ASC) is proposed to combat several security issues like an offline guessing attack, session linking attack, and so forth [74]. Another IoV oriented authentication scheme, Authenticate Key Management (AKM-IoV) protocol is established where the session key is produced for communication between entities after mutual authentication [75]. Modification of Conditional Privacy Preservation Authentication (CPPA) is proposed for an intelligent transport system known as the Pairingless Modified Efficient Conditional Privacy Preservation Authentication Scheme (PME-CPAS) [76], where the pairing mechanism is ignored. The artificial intelligence-based DL approach is an efficient mechanism for identifying data characteristics and then applying a holistic authentication protocol for enhancing the system's security [77].

3) Blockchain for Security: Blockchain, coined from the concept of bitcoin, is the most recent security method adopted in the field of communication. For establishing secure communication between sender to receiver, the sender sends the data, which is considered the block, is broadcasted to every trusted node to the network [78]. The block is then added to the chain to reach the destination. Blockchain holds the capability to fulfil the triad concept of security issues [79]. Blockchain for IoT, named Blockchain of Things (BCOT), is brought forward where edge devices contain the hash of Blockchain for carrying through the cloud via fog [80]. IoTChain is another blockchain-based security architecture, which is derived from the combination of Object Security Architecture (OSCAR) and Authorization for Constrained Environment (ACE) framework [81]. However, the Blockchain methods holding the idea of Direct Acyclic Graph (DAG) based on consensus mechanism, where IoT is introduced for service enhancement [82]. Also, intelligent wireless networks are mingling the concept of AI and Blockchain, which gives a new dimension to security systems [83]. Another novel security-oriented Blockchain framework proposed for SDN enables 5G VANETs to utilize all network nodes and efficiently enhance the security system for the automotive sector [84].

4) Encryption for Security: Encryption is one of the widely used security measures for modern-day technologies for its potentiality. A lightweight attribute-based encryption system named LightWeight Revocable Hierarchical Attribute-based Encryption (LW-RHABE) is proposed by [85] for an IoT environment, where attribute-based encryption allows us to send data to several authorized users without learning their public key. Another Attribute-based encryption algorithm, Ciphertext Policy Attributed-based Encryption (CP-ABE) proposed in [86]. Homomorphic encryption allows third-party entities (cloud, service providers) to execute some computation on encrypted data, which are presented in different works of literature with different varieties known as Fast Fully Homomorphic Encryption (TFHE) [87] and nGraph-HE [88]. On the other hand, Identity-based encryption, namely Identity-based Fully Homomorphic Encryption (IBFHE) [89] and Identitybased Encryption with Authorized Equivalence Test (IBEAET) for surfacing cloud IoT, which encrypted only targeted data [90]. Besides, the Cryptographic Mix zone (CMIX) is a significant tool for designing vehicular networks to ensure desired security. In this case, A security protocol stands on the CMIX concept, One Time Identity based Authenticated Asymmetric Group Key Agreement (OTIBAAGKA) is also proposed to enhance the security of vehicular communication systems [91]. 
TABLE III

COMPARATIVE ANALYSIS ON SECURITY

\begin{tabular}{|c|c|c|c|c|c|}
\hline Features of Security & Access Control & Authentication & Blockchain & Encryption & Intrusion Detection System \\
\hline Implementation Mode & Centralized 95 & $\begin{array}{l}\text { Centralized/ Decentralize } \\
{[75],[76]}\end{array}$ & $\begin{array}{l}\text { Centralized/ } \\
\text { Decentralized } 96\end{array}$ & $\begin{array}{l}\text { Centralized/ Decentral- } \\
\text { ized } 79\end{array}$ & $\begin{array}{l}\text { Centralized/ Decentralized } \\
{[94}\end{array}$ \\
\hline Data Encoding & Not applicable & Not applicable & Applicable $[96]$ & Applicable 79 & Not applicable \\
\hline Attack Protection & Fully protective $[70]$ & Fully protective & Partially Protective 80 & Not Protective & Fully protective \\
\hline Incorporating Intelligence & Not implemented & Implemented 77 & Implemented 83 & Implemented [79] & Implemented [97] \\
\hline Satisfying Security Triad & Partially & Partially & Fully |79 & Partially & Partially \\
\hline $\begin{array}{l}\text { Computational } \\
\text { Requirement }\end{array}$ & Moderate $\mid 71$ & Moderate $\mid 74$ & High 79 & High [79] & Moderate [92] \\
\hline
\end{tabular}

a) Intrusion Detection System for Security: An intrusion Detection System (IDS) is an integral part of defending the system against all kinds of attacks. CNN-based IDS system (CNN-IDS) trained the system and constituted a model to prevent attacks [92]. Also, DL approached Restricted Boltzman machine-based clustered IDS (RBC-IDS) has come into the field of IDS for WSN [93]. Another novel, data-driven Deep CNN-based IDS proposed in [94] to ensure the security information security by detecting traffic flows irregular fluctuations for IoV.

5) Comparative Analysis of Security: The security issue is a grave concern for every organization, every system in the current world. The coverage of security issues is huge and multifarious, which makes it unbearable to gather all features under an umbrella. Keeping the eyesight solely on VHMS security on the basis of the above review, Table III sheds light on some common features comparison studies. Firstly, the implementation mode has the top priority to enforce the security for VHMS. The working principle of Blockchain has the privilege to implement in a decentralized fashion. The device or any other node can generate a hash function, which makes it distinctive over other security techniques. Data encoding capability can be treated as an important feature where no other security measures ensure data protectiveness stronger than encoding. Attack mitigation is one of the prime requirements of the modern data system. The diverse application and heterogeneous nature of devices open up opportunities for multiple types of attacks. Moreover, craving valuable financial, personal, and business transactions data, the malicious and poisonous attacks are staged by hackers substantially. The rigidness against attacks is also necessary for the smooth operation of the system.

\section{E. Interoperability of VHMS}

The interoperability concept was introduced for VHMS so that other existing wireless systems such as GSM, LTE, 5G, and so forth can utilize as the resources of VHMS. It also opens the door for financial profit. Since interoperability is impossible without co-existence, in this literature, interoperability and co-existence are used interchangeably. Interoperability facilitates a system's interfaces to work with other systems where the system may not face any unwanted limitation while other systems using that system's resources.

1) Interoperable $5 G$ : The interoperability of $5 \mathrm{G}$ with LTE and Wi-Fi is improved by the properly isolated antenna, which is studied in [98]. On the other hand, the interoperability of cellular V2X and Ad Hoc vehicular networks of $5 \mathrm{G}$ is overcome by designing an energy sensing-based spectrum sharing scheme [99], [100]. Also, the co-existence of two frequency bands, i.e., $28 \mathrm{GHz}$ and $70 \mathrm{GHz}$ in $5 \mathrm{G}$ are used to operate the system smoothly while minimizing the interference [101].

2) Interoperable Wi-Fi and LTE: The interoperability issue between Wi-Fi and LTE is solved by applying the decoupling method where both systems' co-existence is possible by allocating resources in adaptive spectrum partition fashion [102]. A CNN-based approach that identifies Wi-Fi and LTE through learning in an unlicensed spectrum enhances the co-existence by ensuring interoperability [103].

3) Interoperable TVWS: For interoperable TVWS, a hierarchical model for spectrum sharing is proposed for the coexistence of a heterogeneous network where a fixed user can rent spectrum from tv broadcaster and share the spectrum with the mobile user in the vicinity [104]. Another busy tone-based algorithm for IEEE 802.af and IEEE 802.22 is emerged by proposing in [105] to coexist in the TVWS band.

4) Interoperable Multiple Systems: According to [106], Explicit Channel Coordination (ECC) is a viable technique for enhancing the interoperability of multiple systems using the same spectrum. Besides this, efficiently handling interoperability for multiple systems, a favorable data delivery technique known as Statistical Signal Transmission is proposed by [107].

5) Comparative Analysis of Interoperability: The interoperability concept is widely accepted and introduced in common systems like Wi-Fi, LTE, etc. However, the priority should be made on interfaces to create a system interoperable so that systems can exchange data among them efficiently. Besides, to make this feasible, the radio parameters like spectrum availability could be checked first.

\section{F. Application of VHMS}

The VHMS's significance is rising tremendously with changing times, and future application scope is expanding exponentially. However, some possible and promising application scopes are described below to keep the automotive sector a step ahead.

1) Intelligent Transportation System: Since VHMS isn't a new concept, but the incorporation of different sensors and actuators with advanced technologies facilitated a new scope for the Intelligent Transport System (ITS) to minimize fuel consumption, road accidents, proper travel time utilization, and smooth national economic growth [108]. In this case, the concept of secured and intelligent VHMS framework may enrich the central ITS outstandingly by ensuring excellent 
emergency vehicle (EV) management [109], traffic signal management, street light management, stolen vehicle recognition [110], traffic rules disobeyed vehicle recognition, instructive driving [111] and so forth through big data analytics.

2) Smart City: A fast, intellectual, reliable, and highly secure system development is too important in the smart city for the enriched user-friendly vehicle management infrastructure by connecting all vehicles, various service facilities, different devices, and a smooth parking system. Because haphazard traffic movement and lacking real-time parking information are lead to the possibility of traffic accidents, environmental pollution, waste of valuable time, waste of money [112], and many other inconveniences. To overcome such circumstances and make a hassle-free smart city, the employment of this secure and intelligent VHMS framework with big data analytics may open a new threshold to share and receive the required necessary information among vehicle users, central city traffic management authority, and other bureaucratic organizations.

3) Autonomous Driving: There are lots of research on autonomous vehicles today to make a relaxed, comfortable, secure, and reliable journey by reducing traffic accident and ensuring the highest safety for the road users [113]. Besides, IoV technology's combination with an autonomous vehicle enriched the autonomous driving system remotely [114]. But practically minimal research or application appears by using that technology to share and receive between physically driven and driverless vehicles to make the road safest for travelers when these vehicles are operating over the road. However, the concept of a secure and intelligent VHMS framework using big data analytics may advance and ideally enrich the automotive sectors to operate and monitor autonomous vehicles with manually driven vehicles centrally.

4) Platoon-Based Driving: Platoon-based driving is a technique to drive the vehicle on the highway in a close-flowing group manner to ensure safety and traffic efficiency at the same speed for a massive traffic throughput. Its primary purpose is to establish cooperation among vehicles with common interests like passenger safety, improving traffic efficiency, reducing vehicle emissions, and fuel consumption due to air drag reduction [115]. However, in central VHMS, the stakeholders can share their information and arrange the vehicle platoons as road trains from different regions using big data analytic techniques. In this case, heavy-duty vehicles, besides other vehicles, might be benefited from this technique's employment as a fast, reliable, and secure system [116].

5) Intelligent Vehicle Monitoring: Currently, efficient vehicle monitoring is a big issue worldwide, and gradually it expands to broaden the beneficial aspect in different matters that relate to the automotive industry. An intelligent system for vehicle monitoring matters is capable of observing the vehicle infrastructure status as defined issues to provide an instant decision and instruction to overcome any unexpected incident by using critical thinking, self-learning, and problem-solving developed logic [14]. In this case, the central VHMS framework concept may integrate these features more sophisticatedly to ensure the first, reliable, and accurate data analysis of collected data. Besides, this framework is also enriched by incorporating secure information sharing and receiving facilities among the different stakeholders for a massive number of vehicles using big-data analytic techniques.

6) Telematic Features: Since the number of vehicles is increasing dramatically. As a result, the importance of telematics is growing day by day to ensure vehicle users' safety, security, and comfort [6]. So, the users' vehicles could be equipped their vehicles with the necessary devices for ride-sharing purposes [117], forensic purposes [6], essential evidence of insurance purposes [118], and so forth. These matters could be handled efficiently using an IoE-based secure and intelligent system with big-data analytics in the central entity.

7) Vehicle Observation by Stakeholders: Here, the stakeholders are defined as the different organizations and individual personnel like governmental organizations, vehicular manufacturers, vehicle owners, vehicle drivers, law enforcement agents, insurance agents, ride-sharing organizations, and many others that relate to it. In this case, extending the central VHMS framework's application depicted that vehicular structural information and its performance details, maintenance history, vehicle driver, owner details, vehicle brand information, and performance details could be available under an umbrella. From where the individual stakeholders can collect and compare the required info for further decisions and necessary measures. For example, the government can monitor driver activity, the purpose of vehicular use, traffic management system, individual brand performance for future market acceptance, and so forth.

8) Environmental Pollution Monitoring: Presently, the environmental issue is a big concerning matter. Running vehicles are 12 to 70 percent responsible for polluting air by emitting different gasses and particulates [119], threatening our existence in the world. Currently, to prevent air pollution, various procedures and methods are developed to monitor and control vehicles' emissions. But these are planned to apply in individual vehicles or specific insignificant areas [120]. We framed this secure and intelligent VHMS for the central application nationwide to strengthen and enrich these vehicular emission monitoring and control features.

9) Emergency Assistance: The significance of handling emergency vehicles is enormous in our daily lives because minor ignorance may lead to a devastating situation. It might happen due to individual and limited network capacity constraints on fire brigade vans, ambulances, police vans [121], emergency rescue facilities contained vans [122], road condition monitoring systems [123], and many others. But the concept of the VHMS framework is capable of mitigating these issues centrally with fast, secure, and intelligent ways using big data analytics techniques.

\section{SECURE AND InTELLIGENT Framework FOR VHMS EXPLOITING BIG-DATA ANALYTIC}

This conceptual framework's benefits are massive because it can diagnose vehicular health and deliver reports intelligently from any position besides ensuring the stakeholder's security. This idea could also help develop an intelligent VHMS that is envisioned to reduce the number of fatalities from road accidents by instructive driving and prompt maintenance activity. Besides, reduce maintenance expenses, curb environmental 


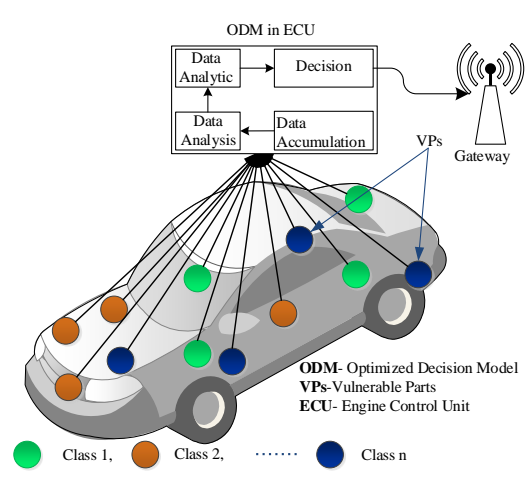

Fig. 3. Conceptual Idea of ODM for VHMS of Individual Vehicle.

pollution, promote travel safety and comfort to the road users, provide convenient access to the stakeholders for a comprehensive set of vehicular and road data information. Government can get real-time vehicle movement information and the exact quantity of operable vehicles. Minimize the vehicle's burglary and its abuse. The manufacturer can get an idea about the movement of their current vehicles, identify the marketed dropout reasons, forecast future demand, and improve quality by observing real-time vehicle-specific model performances and so forth. However, the framework of secure and intelligent VHMS is described as follows.

\section{A. Optimized Decision Model for VHMS}

The development of the Optimized Decision Model (ODM) framework for VHMS may allow the driver, owners, and other stakeholders to learn about the vehicle's real-time behavior. Currently, machine-learning algorithm employment is increasing outstandingly to provide more precise, reliable, and robust decisions about irregularities in real-time. Similarly, this framework has an excellent prospect for performing an accurate and precise analytic process for vehicle fault diagnosis [124]. As a result, this system may reduce maintenance costs by predicting vehicle irregularities, providing instructive driving, pedestrian safety, passengers' comfortability, safety, security, preventing accidents by real-time decision-making. Also, hoping that this idea may serve as a guideline to develop an advanced VHMS in real-time for future studies in the automotive sector. However, the overview of VHMS is represented in Figure 3, and the framework of ODM development for VHMS is discussed systematically.

1) Identify and Correlate the Vulnerable Matters with ODM for VHMS: Primarily need to identify the vulnerable matters which influence vehicular performance and passenger safety, security, comfortability, pedestrian safety, and so forth to correlate these matters with ODM. Because the vehicle has some sensitive items or matters that influence the aforementioned issues, for mitigating these issues, the vulnerable components need to be identified by studying individual vehicular parts requirements and examining their critical functions. In this case, the respected researcher may consider some specific elements as identification parameters, which may help to select actual vulnerable items from lots of vehicular items, i.e., the amount of probable damage, loss, and the parts' importance

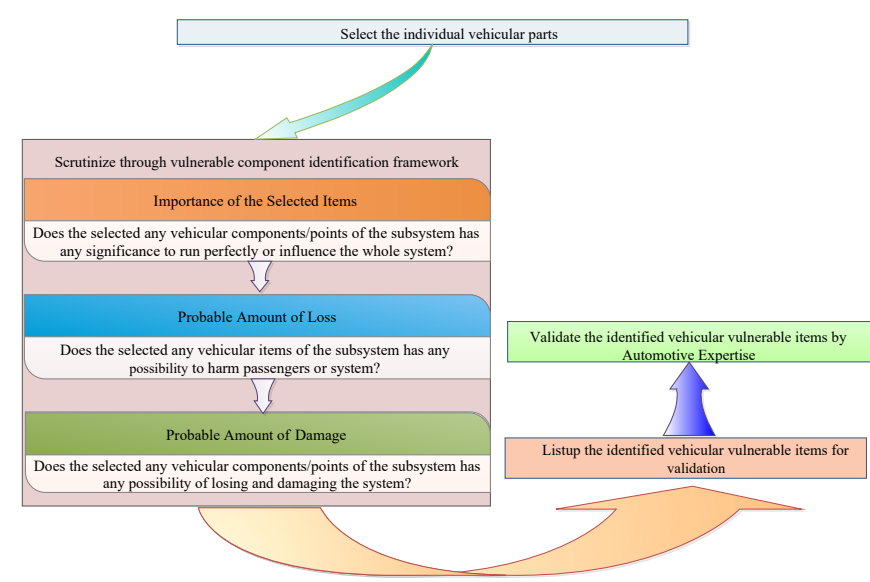

Fig. 4. Conceptual Idea of Vehicular Vulnerable Parts Identification Framework.

and impact on vehicular performance, etc. To do this, they can examine practically the individual vehicle components and study them thoroughly to find the specific reason that affects vehicle performance, vehicle health, passenger safety, and road users' safety (Figure (4). However, this activity may be performed clusters-wise or zone-wise as a subsystem. Furthermore, the identified vehicular vulnerable parts may be validated by consultation and validation from automotive expertise. Afterwards, validating the identified vulnerable items could help to correlate them to develop ODM.

2) Categorization of Identified Vehicular Vulnerable Items: Since the vulnerable items significantly impact the vehicular performance, and if the identified item is appropriately categorized, it may help calculate the risk factor for developing perfect ODM. The value of risk factors may manipulate the priority decision about the maintenance of faulty vehicular items outstandingly. In this case, the researcher may prepare the questionnaire table and may classify the vehicle's vulnerable items by following that table. For example, how it affects the passengers and road users, how much adverse impact on the internal vehicle parts or other resources, and how much risk is associated with it when the irregularities occur. After categorizing these vulnerable items and determining the risk factors, the researcher may utilize these to develop the ODM for accurate fault detection decisions.

3) ODM Development and Performance Evaluation: In this phase, the researchers may utilize the irregularity information of respective vulnerable items' beside the calculated risk factors to develop ODM for achieving robust and accurate decisions in real-time. For that, the researchers need to install sensors and actuators respective to the vulnerable parts to sense the required information against the irregularities. For networking and communication inside the vehicle, Intra-vehicular communication (IVC) is a suitable option with the IoE technology. After that, the efficient analytic process is essential for the real-time decision exploiting accumulated data from the vehicle. We also presumed that vehicles might have many vulnerable parts from our practical experiences and generate 
tons of data from these vulnerable items in every second. So, to maintain massive data and create instant firm decisions, the researcher may employ a big-data analytic approach with ML algorithm techniques. The model's performance evaluation can ensure whether the developed ODM works appropriately or not for vehicular fault diagnosis. Subsequently, it may notify the driver about the vehicular health details using the built-in vehicle notification system and display. In addition, the researcher may consider a V-to-I (Vehicle to Interface) communication network (can be ensured by Subsection III-B) for delivering the decision about the vehicular health to the installed Sink (Fog) using the data handover zone (for example, traffic light, toll-plaza, and residence) to share with other stakeholders. Finally, this ODM can monitor vehicle health in real-time for excellent vehicle longevity with minimum maintenance. Besides advancing transportation management to reduce road accidents, develop smart cities, and minimize pollution for a safe and pleasant journey. Another notable matter is that all sensors and actuators may not be able to transmit data together and need to reduce data traffic load. Therefore, the congestion of the network must need to be diminished, and for this matter, the hybrid Mac (by combining History and Priority approach) strategy is a good option [125].

\section{B. IoE-Driven Multi-Tier HetNet for VHMS}

For enhancing VHMS, the IoE is an emerging concept that can oversee the vehicles' health status and potential risks besides storing the associated information in a central entity, which the stakeholders can further access and utilize. The stakeholders are Drivers, Owners, Government-Organizations or Agencies, Automotive Industries, Non-government Organizations Agencies with varying interests. In this case, there are great possibilities for devising an effective and efficient network-enabled VHMS to ensure different users' access from various networks. So, the scientists can develop the vehicular-communication platform with an excellent vehiculardata collection approach and the data-analytics technique, where heterogeneity might be a prime concern for such a system. However, the IoE-Driven Multi-Tier Heterogeneous Networks (HetNets) conceptual idea for VHMS is presented in Figure 5 and described below eloquently.

1) Multi-Tier Heterogeneous Network: Previously, we discussed that the beneficiary stakeholders might connect or new users willing to join in central VHMS from the different networks at any time from anywhere. A flexible networking system needs to develop for VHMS, where the stakeholders (e.g., Drivers, Owners, Government Organizations, Automotive Industries, Insurance Agencies) can utilize the vehicular structural information and traffic information by the Internet of Everything (IoE) technology from various networks. In this case, the respected researchers may consider IoE-driven heterogeneous networks to ensure sound network communication systems for central VHMS. For the excellent network in central VHMS, they may employ IoE-IVC (Internet of Everything- Intra-vehicular Communication), V-to-I (Vehicle to Interface), and I-to-C (Interface to Cloud) communication system. Since this concept proposes bringing all vehicles of a

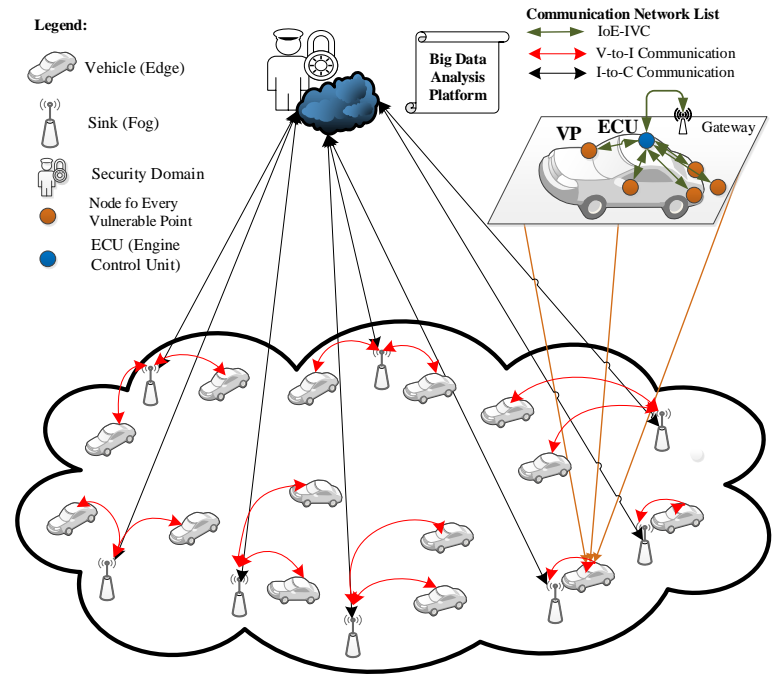

Fig. 5. Conceptual Idea of IoE-Driven Multi-Tier HetNet for VHMS.

nation under an umbrella, there could be a more significant number of vehicles and may produce tons of data every second. For this condition, we suggested that the respected research may consider a Multi-Tier Heterogeneous Networks (Multi-Tier HetNet). So that, it can build a robust vehicular communication platform for VHMS by using various sets of base stations (BSs) like Macrocell, Picocell, Femtocell for the massive number of vehicle connectivity to share their vehicle health status and other information (telematics) during their movement [126].

Moreover, the scientist may design the HetNet architecture for VHMS by incorporating all the homogeneous networks. Different kinds of connectivity technologies (i.e., Zigbee, WiFi Gateway, NB-IoT(LTE), Cellular Technology, TV White Space, and so on) can be used in different levels to collect data from the various vehicles by following Subsection III-A Therefore, a software-defined platform may be considered here for handling the collected data from the different networks with and without the presence of the cellular coverage area.

2) Data Handling Mechanism: In central VHMS, MultiTier HetNet can deliver a massive amount of information from the colossal number of vehicles, and for this robust and efficient data handling technique needs to process and extract concealed information from large datasets. Also, it is not essential to store all the data on the server, and instead of that, only crucial data should be stored to reduce the system's unnecessary data traffic. Again, due to the different network nature and massive data, incorporating the conventional data handling approach may not be feasible for central VHMS. So, we advise the respected researchers to develop an efficient data handling mechanism. However, ML-enabled big-data analytics are capable of reducing a considerable portion of storage data with maximum analyzing accuracy [127]. In this case, ML has high precision, high performance, and high scalability as a bigdata handling mechanism than the hybrid, cloud-based, agentbased, and heuristic-based mechanisms [128]. Therefore, the researchers may use this technique for storing essential data 


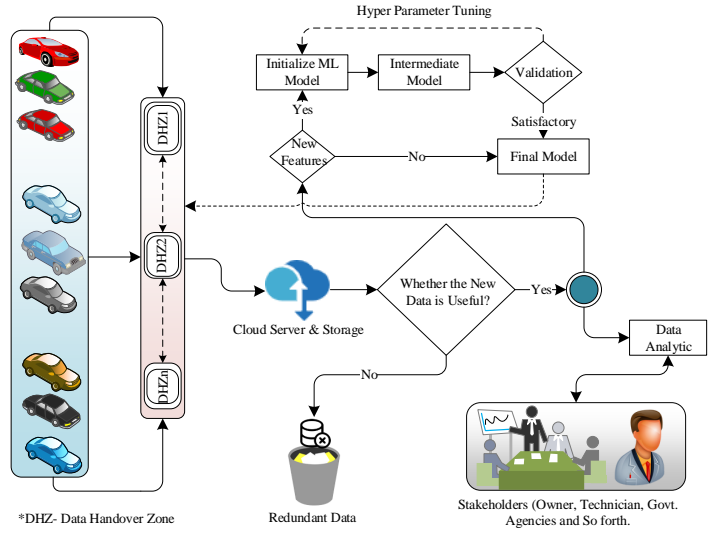

Fig. 6. Conceptual Idea of Data Analytic Process in Cloud.

instead of insignificant data because this can reduce the networks' traffic load. In this, they may utilize "Big Data Reduction during Data acquisition" on edge by collecting only necessary vehicular data and "Big Data Reduction through Decision Scheme" at Fog and Cloud by reducing data after some pre-processing activity.

3) Data Analytic Process for Central VHMS: In subsection III-A, we discussed the data analytic process, which is utterly different from this phase because it is only responsible for performing this process inside the vehicle on the collected sensor-actuator data. For central VHMS to execute the data analytic process at the cloud, data may come from different Data Hanover Zone to store in cloud storage. From there, the data can be pulled for making ML models and finding insights. However, there might be a possibility of receiving redundant data while pulling through queries. In this case, the researcher may set a decision engine to monitor this process. If the data is identified as redundant, it could be dropped down automatically to the trash. They may consider two sections where the new data might be processed. One is for developing machine learning models, and another one is for analyzing data and finding insights. If they want to evaluate this analytic process's performance, they can create a machine learning model using the new data in the machine learning section as per Figure 6 Repeatedly the model's performance might be evaluated until it reaches the desired outcome by following well-known metrics. After passing the evaluation step, the data analytic process may continue. If it finds insights and then submits reports to the stakeholders according to their requirements, and may store them for future references.

\section{Service Aware Trustworthy Networks for VHMS}

As an emerging technology, IoE can play an essential role in the VHMS of the automotive industry by integrating the stakeholders, process, data, and things via vehicular communication networks, where security is one of the prime concerns against the cyber-attacks. We depicted this concept to encourage the researchers for future research on secure VHMS by ensuring Data and Networks-Security (DNS). Since this central VHMS idea included the Heterogeneity and Scalability issue, the respected researchers may consider the network's trustwor-

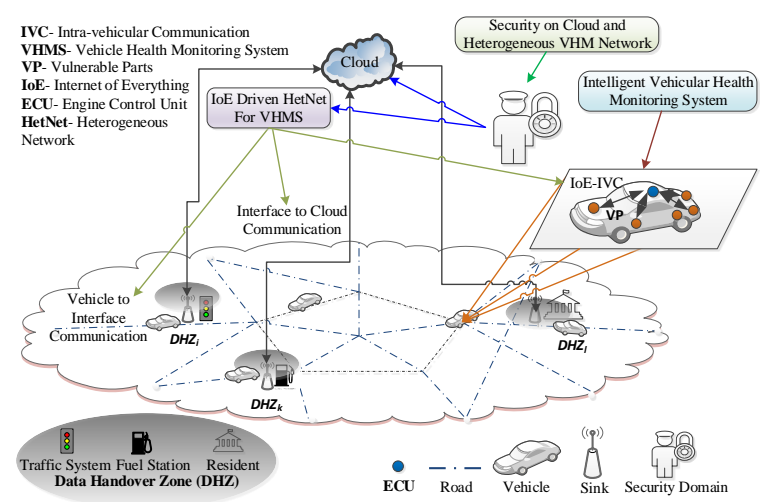

Fig. 7. Conceptual Idea of Service Aware Trustworthy HetNet for VHMS

thiness, secure data collection, and data integrity matter for DNS in VHMS. However, for designing and developing secure and trustworthy networks for VHMS, the conceptual idea is presented in Figure 7 and described systematically in bellow.

Since this idea covers a large extent in the automotive sector, to ensure a better and hassle-free facility, the researcher should keep in mind QoS in VHMS. Also, secure the collected data of individual vehicular vulnerable parts, ensure confidentiality, data integrity, and assure a trustworthy network for stakeholders. For secure QoS-aware VHMS, they can apply the software-defined technique to classify the requested services from the stakeholders and provide service according to the service requirements. Besides, the accumulated data need to be secure and protected from threats, intrusion attacks and avoids data compromised. In this case, they may focus on the cryptosystem and secret sharing by distributing storage data in several cloud servers using SlepianWolf coding (SWC) because it is more efficient in terms of storage, communication, and computation cost. This distributed storage can avoid attackers and secure the data.

Furthermore, we advise the researcher to employ the lightweight Intrusion Detection System (IDS) as a promising solution for developing a trustworthy network using an ML approach. Finally, the researcher may ensure data integrity, accountability, authenticity, confidentiality during the data transaction by encrypting data exploiting blockchain technology. It can help secure data integrity even though the attacker attacks the data, but data will make no sense.

\section{Open Research Scope for Central VHMS}

Day by day, the significance of vehicular components and performance monitoring is increasing marvelously. Recently, these systems have been upgraded as a vehicle health monitoring system (VHMS) to monitor the vehicular operation and its essential elements remotely compared to OBD II using smart devices and technology. It's a matter of time to develop central VHMS by enhancing this matter to reduce the fatality rate due to road accidents, reduce maintenance expenses, improve vehicle life, improve safety, passengers, and pedestrians' safety. However, for developing a robust and 
efficient central VHMS, there are many challenges and future research scope described as follows.

\section{A. Profound Study on Vehicular Parts}

Usually, the vehicle comprises thousands of elements and parts, but they are not the same priority item due to their individual and unique functionalities. Some of them perform prime responsibility during the operational times and maintain the vehicular condition satisfactorily besides better services. But throughout our literature study, we found that previous research work only discussed the functionalities of essential vehicular elements and their significance on vehicular performance. Merely these matters don't carry importance to built a central VHMS, and till today, there is no mathematical progress to prioritize these vital elements for utilizing in this monitoring system. So, we think there is a massive possibility of researching vehicular parts to develop a mathematical model for prioritizing the vehicular components, which may help develop the central VHMS.

\section{B. Well-organized Collaborative Data-exchange Platform for Central VHMS}

Day by day, the number of connected vehicles is increasing dramatically, so the possibility of remote monitoring of these is growing similarly. Besides that, the importance of VHMS is also rising for monitoring vehicular performance and operations remotely. We noticed that it has application scope to a central extent for various beneficial aspects. But the problem about the existing network, which has lots of limitations to connect the massive number of vehicles with roadside units, installed devices, surveillance systems, intelligent traffic systems, cloud storage and sever systems, and so forth. For that, central VHMS requires a well-organized and efficient multi-tire communication network system to ensure a robust vehicular communication platform for big data collection and data analytics as a collaborative data exchange platform using IoT technology. In this case, Multitier Heterogeneous Network is a good option and can serve the required demand efficiently. There is an excellent research scope to develop an efficient and well-organized network for central VHMS to interconnect different links and nodes as a common platform.

\section{Cyber Security of Central VHMS}

In central VHMS, the uncountable number of stakeholders besides the uncountable vehicles, devices, and so forth might be connected. In this case, they can share their personal information, vehicular information, automobile industry, and government organizational information. These are valuable and must be protected from intruders; otherwise, the whole system may damage within a few seconds and abuse helpful information. For that, it is necessary to restrict intruder access, and that is possible by developing a healthy, secure, and robust networking system by developing unique techniques using Blockchain technology. There is a possibility of creating a new scalable VehiChain model exploiting Blockchain technology to establish a central entity's secure vehicular communication network system.

\section{Minimization the Complexity of Users Information Sharing}

For establishing central VHMS, the system should be amiable to all types of users besides the security and services issues. Because in our society, different classes and different opinion people are living and may have different tastes. In this case, the central VHMS should be established so that all categories of people can easily access the system and be user-friendly to them besides ensuring privacy protection. Otherwise, a significant part of them might be loos their interest in using it. To overcome this, during the development of central VHMS, the researcher may conduct further study to make it easier for all people by minimizing the system's complexity.

\section{E. In-depth Study to the Implication of Central VHMS}

The VHMS has a significant influence on improving vehicular performances besides reducing maintenance expenses, accidental rates, improving vehicle longevity, improving pedestrian and passengers safety, comfortability, and so forth. Besides these, for establishing this VHMS to a central extent, no research activity was conducted that revealed these issues or how much it can contribute to national economic growth compared to establishing such a system. However, the researcher can conduct an in-depth study before establishing the ceratin area because it could minimize the project expenses besides enhancing the facility of a central VHMS.

\section{F. Energy Efficient Central VHMS and Managing Alternative Sources}

For the central and individual VHMS context, many sensors, actuators, networking devices, roadside instruments need to install, and these may run continuously. As a result, idle devices and instruments may consume energy and increase operating expenses, though, in various sectors, respected researchers are researching to minimize energy consumption with better efficiency. So, the central and individual VHMS has an outstanding research scope to develop an energy-efficient system using adaptive intelligent energy-efficient analytics machines and devices. On the other hand, sudden power failure of such a system may lead to a massive loss. The respected researcher can conduct in-depth research on developing alternative power sources like green energy sources to overcome this situation.

\section{CONCLUSION}

The importance of the vehicle in our daily lives is massive due to its excellent carrying capabilities, fast and reliable movement, increasing work productivity, contribution to national economic growth, and so forth. But unexpected vehicular downtime wastes our valuable time and money, pollutes the environment, and people become disabled for reckless driving and die from severe accidents and so forth. Based on the current perspective and demand, it is necessary to enhance the vehicle with advanced and intelligent features to mitigate these issues. Thus, the intelligent VHMS features are a prominent and promising matter in the current perspective to improve vehicle performances, longevity, instructive decision, 
secure and comfortable journey, and many others. Based on these benefits, it seems that the central VHMS has massive benefits by bringing all operational vehicles under surveillance systems. For that, we proposed in detail a safe and intelligent central VHMS framework with a multi-tire HetNet system to give the direction for future research work and move ahead of the automotive industry. Before that, the proposed framework makes it more understandable by proposing the taxonomy of VHMS as the preliminary glimpse of central VHMS. At the end of this article, we also discussed future research scope eloquently for inspiring the researcher to enhance the central VHMS with more sophisticated technology and features. Anticipating that, the stakeholders could be benefitted individually in enormous ways and bring the governmental organization besides the vehicle manufacturers and different organizations under the same umbrella. Conclusively, this article may act as a complete guide to develop enhanced VHMS for the automotive sector and inspire the researcher to establish perfect real-time VHMS for the central entity.

\section{REFERENCES}

[1] M. El Zorkany, A. Yasser, and A. I. Galal, "Vehicle to vehicle "v2v" communication: Scope, importance, challenges, research directions and future," The Open Transportation Journal, vol. 14, no. 1, 2020.

[2] C. Yu, J. Li, X. Li, X. Ren, and B. B. Gupta, "Four-image encryption scheme based on quaternion fresnel transform, chaos and computer generated hologram," Multimedia Tools and Applications, vol. 77, no. 4, pp. 4585-4608, 2018.

[3] F. Jia, Y. Lei, L. Guo, J. Lin, and S. Xing, "A neural network constructed by deep learning technique and its application to intelligent fault diagnosis of machines," Neurocomputing, vol. 272, pp. 619-628, 2018

[4] B. Tang, Z. Chen, G. Hefferman, S. Pei, T. Wei, H. He, and Q. Yang, "Incorporating intelligence in fog computing for big data analysis in smart cities," IEEE Transactions on Industrial informatics, vol. 13, no. 5, pp. 2140-2150, 2017.

[5] A. J. M. Muzahid, S. F. Kamarulzaman, and M. A. Rahim, "Learningbased conceptual framework for threat assessment of multiple vehicle collision in autonomous driving," in 2020 Emerging Technology in Computing, Communication and Electronics (ETCCE). IEEE, 2020, pp. 1-6.

[6] I. Cvitić, D. Peraković, M. Perisa, and S. Husnjak, "Application possibilities of digital forensic procedures in vehicle telematics systems," Zeszyty Naukowe Wyższej Szkoty Technicznej w Katowicach, 2018.

[7] S. Chavhan, D. Gupta, C. Nagaraju, A. Rammohan, A. Khanna, and J. J. Rodrigues, "An efficient context-aware vehicle incidents route service management for intelligent transport system," IEEE Systems Journal, 2021.

[8] A. Al-Qerem, M. Alauthman, A. Almomani, and B. Gupta, "Iot transaction processing through cooperative concurrency control on fogcloud computing environment," Soft Computing, vol. 24, no. 8, pp. 5695-5711, 2020.

[9] G. Hinge, R. Y. Surampalli, M. K. Goyal, B. B. Gupta, and X. Chang, "Soil carbon and its associate resilience using big data analytics: For food security and environmental management," Technological Forecasting and Social Change, vol. 169, p. 120823, 2021.

[10] J. Ali, M. A. Rahman, M. Z. A. Bhuiyan, A. T. Asyhari, and M. N. Kabir, "Cyber-physical autonomous vehicular system (cavs): A mac layer perspective," in Big Data Analytics for Cyber-Physical Systems. Springer, 2020, pp. 129-152.

[11] S. u. Rehman, M. Ceglia, S. Siddiqui, and V. Gruhn, "Towards an importance of security for cyber-physical systems/internet-of-things," in Proceedings of the 2019 8th International Conference on Software and Information Engineering, 2019, pp. 151-155.

[12] Z. El-Rewini, K. Sadatsharan, N. Sugunaraj, D. F. Selvaraj, S. J. Plathottam, and P. Ranganathan, "Cybersecurity attacks in vehicular sensors," IEEE Sensors Journal, vol. 20, no. 22, pp. 13752-13767, 2020
[13] N. M. Sai, B. V. Prakash, N. Renuka, K. Deepu, and B. Jayanag, "Vehicle monitoring system," International Journal of Recent Technology and Engineering, vol. 8, no. 6, pp. 5212-5215, 2020.

[14] U. Shafi, A. Safi, A. R. Shahid, S. Ziauddin, and M. Q. Saleem, "Vehicle remote health monitoring and prognostic maintenance system," Journal of advanced transportation, vol. 2018, 2018.

[15] H. Wang, Z. Li, Y. Li, B. B. Gupta, and C. Choi, "Visual saliency guided complex image retrieval," Pattern Recognition Letters, vol. 130 , pp. 64-72, 2020

[16] H. Ye, L. Liang, G. Y. Li, J. Kim, L. Lu, and M. Wu, "Machine learning for vehicular networks: Recent advances and application examples," ieee vehicular technology magazine, vol. 13, no. 2, pp. 94-101, 2018.

[17] M. Rahim, M. Rahman, M. A. Rahman, A. J. M. Muzahid, S. F. Kamarulzaman et al., "A framework of iot-enabled vehicular noise intensity monitoring system for smart city," in International Conference on Innovative Technology, Engineering and Science. Springer, 2020, pp. 194-205.

[18] F. Tang, Y. Kawamoto, N. Kato, and J. Liu, "Future intelligent and secure vehicular network toward 6g: Machine-learning approaches," Proceedings of the IEEE, vol. 108, no. 2, pp. 292-307, 2019.

[19] N. A. Spielberg, M. Brown, N. R. Kapania, J. C. Kegelman, and J. C. Gerdes, "Neural network vehicle models for high-performance automated driving," Science robotics, vol. 4, no. 28, 2019.

[20] D. Sirohi, N. Kumar, and P. S. Rana, "Convolutional neural networks for 5g-enabled intelligent transportation system: A systematic review," Computer Communications, vol. 153, pp. 459-498, 2020.

[21] X. Wen-juan and L. Jian-feng, "Application of vision sensing technology in urban intelligent traffic control system," in 2018 4th International Conference on Computer and Technology Applications (ICCTA). IEEE, 2018, pp. 74-77.

[22] H. Yang, L. Guan, J. Nan, X. Zhao, Y. Liang, Q. Yao, A. Yu, and J. Zhang, "Intelligent optical network with ai and blockchain," in 2019 18th International Conference on Optical Communications and Networks (ICOCN). IEEE, 2019, pp. 1-3.

[23] R. Gu, Z. Yang, and Y. Ji, "Machine learning for intelligent optical networks: A comprehensive survey," Journal of Network and Computer Applications, vol. 157, p. 102576, 2020.

[24] X. Zhou, M. Sun, G. Y. Li, and B.-H. F. Juang, "Intelligent wireless communications enabled by cognitive radio and machine learning," China Communications, vol. 15, no. 12, pp. 16-48, 2018.

[25] B. Lorenzo, F. J. Gonzalez-Castano, and Y. Fang, "A novel collaborative cognitive dynamic network architecture," IEEE Wireless Communications, vol. 24, no. 1, pp. 74-81, 2017.

[26] Y. Sun, M. Peng, Y. Zhou, Y. Huang, and S. Mao, "Application of machine learning in wireless networks: Key techniques and open issues," IEEE Communications Surveys \& Tutorials, vol. 21, no. 4, pp. 3072-3108, 2019.

[27] Q. Mao, F. Hu, and Q. Hao, "Deep learning for intelligent wireless networks: A comprehensive survey," IEEE Communications Surveys \& Tutorials, vol. 20, no. 4, pp. 2595-2621, 2018.

[28] C. Liaskos, A. Tsioliaridou, S. Nie, A. Pitsillides, S. Ioannidis, and I. Akyildiz, "An interpretable neural network for configuring programmable wireless environments," in 2019 IEEE 20th International Workshop on Signal Processing Advances in Wireless Communications (SPAWC). IEEE, 2019, pp. 1-5.

[29] J. Jagannath, N. Polosky, A. Jagannath, F. Restuccia, and T. Melodia, "Machine learning for wireless communications in the internet of things: A comprehensive survey," Ad Hoc Networks, vol. 93, p. 101913, 2019.

[30] M. Mehrabi, M. Mohammadkarimi, M. Ardakani, and Y. Jing, "A deep learning based channel estimation for high mobility vehicular communications," in 2020 International Conference on Computing, Networking and Communications (ICNC). IEEE, 2020, pp. 338-342.

[31] M. Veres and M. Moussa, "Deep learning for intelligent transportation systems: A survey of emerging trends," IEEE Transactions on Intelligent transportation systems, vol. 21, no. 8, pp. 3152-3168, 2019.

[32] G. Zhu, D. Liu, Y. Du, C. You, J. Zhang, and K. Huang, "Toward an intelligent edge: Wireless communication meets machine learning," IEEE communications magazine, vol. 58, no. 1, pp. 19-25, 2020.

[33] M. Chen, T. Wang, K. Ota, M. Dong, M. Zhao, and A. Liu, "Intelligent resource allocation management for vehicles network: An a3c learning approach," Computer Communications, vol. 151, pp. 485-494, 2020.

[34] T. Zhang, X. Chen, and C. Xu, "Intelligent routing algorithm based on deep belief network for multimedia service in knowledge centric vanets," in 2018 International Conference on Networking and Network Applications (NaNA). IEEE, 2018, pp. 1-6. 
[35] J. Fan, L. Fang, J. Wu, Y. Guo, and Q. Dai, "From brain science to artificial intelligence," Engineering, vol. 6, no. 3, pp. 248-252, 2020.

[36] T. Alsuliman, D. Humaidan, and L. Sliman, "Machine learning and artificial intelligence in the service of medicine: Necessity or potentiality?" Current research in translational medicine, vol. 68 , no. 4, pp 245-251, 2020.

[37] W. Hua, F. Dai, L. Huang, J. Xiong, and G. Gui, "Hero: Human emotions recognition for realizing intelligent internet of things," IEEE Access, vol. 7, pp. 24321-24332, 2019.

[38] A. Binsahaq, T. R. Sheltami, and K. Salah, "A survey on autonomic provisioning and management of qos in sdn networks," IEEE Access, vol. 7, pp. 73 384-73 435, 2019.

[39] A. Bonci, S. Longhi, and M. Pirani, "Rmas architecture for autonomic computing in cyber-physical systems," in IECON 2019-45th Annual Conference of the IEEE Industrial Electronics Society, vol. 1. IEEE, 2019, pp. 2996-3003.

[40] C. L. Stergiou, K. E. Psannis, and B. B. Gupta, "Iot-based big data secure management in the fog over a $6 \mathrm{~g}$ wireless network," IEEE Internet of Things Journal, vol. 8, no. 7, pp. 5164-5171, 2020.

[41] O. Osahon and E. A. Emmanuel, "A wireless network infrastructure architecture for rural communities," International Journal of Computer Science and Information Technology, vol. 9, no. 3, pp. 43-62, 2017.

[42] M. M. Hussain and M. S. Beg, "Using vehicles as fog infrastructures for transportation cyber-physical systems (t-cps): Fog computing for vehicular networks," International Journal of Software Science and Computational Intelligence (IJSSCI), vol. 11, no. 1, pp. 47-69, 2019.

[43] B. B. Gupta, K.-C. Li, V. C. Leung, K. E. Psannis, S. Yamaguchi et al., "Blockchain-assisted secure fine-grained searchable encryption for a cloud-based healthcare cyber-physical system," IEEE/CAA Journal of Automatica Sinica, 2021.

[44] H. Wang, H. Zhao, J. Zhang, D. Ma, J. Li, and J. Wei, "Survey on unmanned aerial vehicle networks: A cyber physical system perspective," IEEE Communications Surveys \& Tutorials, vol. 22, no. 2, pp. 1027-1070, 2019.

[45] H. Fu, Z. Sharif-Khodaei, and M. F. Aliabadi, "An energy-efficient cyber-physical system for wireless on-board aircraft structural health monitoring," Mechanical Systems and Signal Processing, vol. 128, pp. 352-368, 2019.

[46] A. Rebeiro-Hargrave, N. H. Motlagh, S. Varjonen, E. Lagerspetz, P. Nurmi, and S. Tarkoma, "Megasense: Cyber-physical system for real-time urban air quality monitoring," in 2020 15th IEEE Conference on Industrial Electronics and Applications (ICIEA). IEEE, 2020, pp. $1-6$.

[47] A. Arooj, M. S. Farooq, T. Umer, G. Rasool, and B. Wang, "Cyber physical and social networks in iov (cpsn-iov): a multimodal architecture in edge-based networks for optimal route selection using $5 \mathrm{~g}$ technologies," IEEE Access, vol. 8, pp. 33609-33 630, 2020.

[48] C. Li, H. Zhang, T. Zhang, J. Rao, G. Yin et al., "Cyber-physical scheduling for predictable reliability of inter-vehicle communications," IEEE Transactions on Vehicular Technology, vol. 69, no. 4, pp. 41924206, 2020.

[49] A. Tewari and B. B. Gupta, "Secure timestamp-based mutual authentication protocol for iot devices using rfid tags," International Journal on Semantic Web and Information Systems (IJSWIS), vol. 16, no. 3, pp. 20-34, 2020.

[50] B. B. Gupta and M. Quamara, "An overview of internet of things (iot): Architectural aspects, challenges, and protocols," Concurrency and Computation: Practice and Experience, vol. 32, no. 21, p. e4946, 2020.

[51] V. Adat and B. B. Gupta, "Security in internet of things: issues, challenges, taxonomy, and architecture," Telecommunication Systems, vol. 67, no. 3, pp. 423-441, 2018.

[52] A. Verma, S. Prakash, V. Srivastava, A. Kumar, and S. C. Mukhopadhyay, "Sensing, controlling, and iot infrastructure in smart building: a review," IEEE Sensors Journal, vol. 19, no. 20, pp. 9036-9046, 2019.

[53] K. Liu, X. Xu, M. Chen, B. Liu, L. Wu, and V. C. Lee, "A hierarchical architecture for the future internet of vehicles," IEEE Communications Magazine, vol. 57, no. 7, pp. 41-47, 2019.

[54] J. Contreras-Castillo, S. Zeadally, and J. A. Guerrero Ibáñez, "A seven-layered model architecture for internet of vehicles," Journal of Information and Telecommunication, vol. 1, no. 1, pp. 4-22, 2017.

[55] I. U. Din, M. Guizani, S. Hassan, B.-S. Kim, M. K. Khan, M. Atiquzzaman, and S. H. Ahmed, "The internet of things: A review of enabled technologies and future challenges," Ieee Access, vol. 7, pp. 7606-7640, 2018.
[56] W. Ji, B. Liang, Y. Wang, R. Qiu, and Z. Yang, "Crowd v-ioe: Visual internet of everything architecture in ai-driven fog computing," IEEE Wireless Communications, vol. 27, no. 2, pp. 51-57, 2020.

[57] H. Moura, A. R. Alves, J. R. Borges, D. F. Macedo, and M. A. Vieira, "Ethanol: a software-defined wireless networking architecture for ieee 802.11 networks," Computer Communications, vol. 149, pp. 176-188, 2020.

[58] M. T. Abbas, A. Muhammad, and W.-C. Song, "Sd-iov: Sdn enabled routing for internet of vehicles in road-aware approach," Journal of Ambient Intelligence and Humanized Computing, vol. 11, no. 3, pp. $1265-1280,2020$

[59] X. Lin, "Vehicular networking," IEEE Communications Standards Magazine, vol. 1, no. 2, pp. 68-68, 2017.

[60] A. Soua and S. Tohme, "Multi-level sdn with vehicles as fog computing infrastructures: A new integrated architecture for 5g-vanets," in 2018 21st Conference on Innovation in Clouds, Internet and Networks and Workshops (ICIN). IEEE, 2018, pp. 1-8.

[61] P. Micholia, M. Karaliopoulos, I. Koutsopoulos, L. Navarro, R. B. Vias, D. Boucas, M. Michalis, and P. Antoniadis, "Community networks and sustainability: a survey of perceptions, practices, and proposed solutions," IEEE Communications Surveys \& Tutorials, vol. 20, no. 4 pp. 3581-3606, 2018.

[62] A. Darwish and A. E. Hassanien, "Cyber physical systems design, methodology, and integration: the current status and future outlook," Journal of Ambient Intelligence and Humanized Computing, vol. 9, no. 5, pp. 1541-1556, 2018.

[63] T. Addabbo, A. Fort, M. Mugnaini, E. Panzardi, A. Pozzebon, and V. Vignoli, "A city-scale iot architecture for monumental structures monitoring," Measurement, vol. 131, pp. 349-357, 2019.

[64] M. Ayaz, M. Ammad-Uddin, Z. Sharif, A. Mansour, and E.-H. M. Aggoune, "Internet-of-things (iot)-based smart agriculture: Toward making the fields talk," IEEE Access, vol. 7, pp. 129551-129583, 2019.

[65] S. P. Ahuja and N. Wheeler, "Architecture of fog-enabled and cloudenhanced internet of things applications," International Journal of Cloud Applications and Computing (IJCAC), vol. 10, no. 1, pp. 1-10, 2020.

[66] S. M. Karunarathne, M. Dray, L. Popov, M. Butler, C. Pennington, and C. M. Angelopoulos, "A technological framework for data-driven iot systems: Application on landslide monitoring," Computer Communications, vol. 154, pp. 298-312, 2020.

[67] K. Singh and D. S. Tomar, "Architecture, enabling technologies, security and privacy, and applications of internet of things: A survey," in 2018 2nd International Conference on I-SMAC. IEEE, 2018, pp. 642-646.

[68] C. Tipantuña and X. Hesselbach, "Nfv/sdn enabled architecture for efficient adaptive management of renewable and non-renewable energy," IEEE Open Journal of the Communications Society, vol. 1, pp. 357380, 2020.

[69] M. Sain, A. J. Chaudhray, S. Aich, and H. J. Lee, "Security enhancement for access control mechanism in real-time wireless sensor network," in 2019 21st International Conference on Advanced Communication Technology (ICACT). IEEE, 2019, pp. 1135-1142.

[70] M. Uddin, S. Islam, and A. Al-Nemrat, "A dynamic access control model using authorising workflow and task-role-based access control," IEEE Access, vol. 7, pp. 166 676-166 689, 2019.

[71] S. Ding, J. Cao, C. Li, K. Fan, and H. Li, "A novel attribute-based access control scheme using blockchain for iot. ieee access 7 (2019), 38431-38441," 2019.

[72] M. A. Habib, M. Ahmad, S. Jabbar, S. Khalid, J. Chaudhry, K. Saleem, J. J. Rodrigues, and M. S. Khalil, "Security and privacy based access control model for internet of connected vehicles," Future Generation Computer Systems, vol. 97, pp. 687-696, 2019.

[73] S. Nashwan, "Aaa-wsn: Anonymous access authentication scheme for wireless sensor networks in big data environment," Egyptian Informatics Journal, vol. 22, no. 1, pp. 15-26, 2021.

[74] C.-M. Chen, B. Xiang, Y. Liu, and K.-H. Wang, "A secure authentication protocol for internet of vehicles," Ieee Access, vol. 7, pp. 12047 $12057,2019$.

[75] M. Wazid, P. Bagga, A. K. Das, S. Shetty, J. J. Rodrigues, and Y. Park, "Akm-iov: Authenticated key management protocol in fog computing-based internet of vehicles deployment," IEEE Internet of Things Journal, vol. 6, no. 5, pp. 8804-8817, 2019.

[76] F. Alanazi, S. Al-Shareeda, and F. Ozguner, "An efficient cppa scheme for intelligent transportation networks," Pervasive and Mobile Computing, vol. 59, p. 101041, 2019. 
[77] X. Qiu, Z. Du, and X. Sun, "Artificial intelligence-based security authentication: Applications in wireless multimedia networks," IEEE Access, vol. 7, pp. 172 004-172 011, 2019.

[78] E. Christian, F. Massimo, and G. B. Bhooshan, "Blockchain-based authentication and authorization for smart city applications [j]," Information Processing and Management, vol. 58, no. 2, p. 102468, 2021.

[79] M. Warkentin and C. Orgeron, "Using the security triad to assess blockchain technology in public sector applications," International Journal of Information Management, vol. 52, p. 102090, 2020

[80] H. Hui, X. An, H. Wang, W. Ju, H. Yang, H. Gao, and F. Lin, "Survey on blockchain for internet of things." J. Internet Serv. Inf. Secur., vol. 9, no. 2, pp. 1-30, 2019.

[81] O. Alphand, M. Amoretti, T. Claeys, S. Dall'Asta, A. Duda, G. Ferrari, F. Rousseau, B. Tourancheau, L. Veltri, and F. Zanichelli, "Iotchain: A blockchain security architecture for the internet of things," in 2018 IEEE wireless communications and networking conference (WCNC). IEEE, 2018, pp. 1-6.

[82] B. Cao, Y. Li, L. Zhang, L. Zhang, S. Mumtaz, Z. Zhou, and M. Peng, "When internet of things meets blockchain: Challenges in distributed consensus," IEEE Network, vol. 33, no. 6, pp. 133-139, 2019.

[83] Y. Dai, D. Xu, S. Maharjan, Z. Chen, Q. He, and Y. Zhang, "Blockchain and deep reinforcement learning empowered intelligent $5 \mathrm{~g}$ beyond," IEEE network, vol. 33, no. 3, pp. 10-17, 2019.

[84] L. Xie, Y. Ding, H. Yang, and X. Wang, "Blockchain-based secure and trustworthy internet of things in sdn-enabled 5g-vanets," IEEE Access, vol. 7, pp. 56 656-56 666, 2019.

[85] M. Ali, M.-R. Sadeghi, and X. Liu, "Lightweight revocable hierarchical attribute-based encryption for internet of things," IEEE Access, vol. 8 , pp. 23 951-23964, 2020.

[86] J. Li, Y. Zhang, J. Ning, X. Huang, G. S. Poh, and D. Wang, "Attribute based encryption with privacy protection and accountability for cloudiot," IEEE Transactions on Cloud Computing, 2020.

[87] I. Chillotti, N. Gama, M. Georgieva, and M. Izabachène, "Tfhe: fast fully homomorphic encryption over the torus," Journal of Cryptology, vol. 33, no. 1, pp. 34-91, 2020.

[88] F. Boemer, Y. Lao, R. Cammarota, and C. Wierzynski, "ngraph-he: a graph compiler for deep learning on homomorphically encrypted data," in Proceedings of the 16th ACM International Conference on Computing Frontiers, 2019, pp. 3-13.

[89] T. Shen, F. Wang, K. Chen, K. Wang, and B. Li, "Efficient leveled (multi) identity-based fully homomorphic encryption schemes," IEEE Access, vol. 7, pp. 79299-79310, 2019.

[90] R. Elhabob, Y. Zhao, N. Eltayieb, A. M. Abdelgader, and H. Xiong, "Identity-based encryption with authorized equivalence test for cloudassisted iot," Cluster Computing, vol. 23, no. 2, pp. 1085-1101, 2020.

[91] L. Zhang, "Otibaagka: A new security tool for cryptographic mix-zone establishment in vehicular ad hoc networks," IEEE Transactions on Information Forensics and Security, vol. 12, no. 12, pp. 2998-3010, 2017.

[92] Y. Xiao, C. Xing, T. Zhang, and Z. Zhao, "An intrusion detection model based on feature reduction and convolutional neural networks," IEEE Access, vol. 7, pp. 42 210-42219, 2019.

[93] S. Otoum, B. Kantarci, and H. T. Mouftah, "On the feasibility of deep learning in sensor network intrusion detection," IEEE Networking Letters, vol. 1, no. 2, pp. 68-71, 2019.

[94] L. Nie, Z. Ning, X. Wang, X. Hu, J. Cheng, and Y. Li, "Datadriven intrusion detection for intelligent internet of vehicles: A deep convolutional neural network-based method," IEEE Transactions on Network Science and Engineering, vol. 7, no. 4, pp. 2219-2230, 2020

[95] K. Riad, R. Hamza, and H. Yan, "Sensitive and energetic iot access control for managing cloud electronic health records," IEEE Access, vol. 7, pp. 86384-86393, 2019.

[96] A. A. Abbood, G. Sulong, A. A. A. Razzaq, and S. U. Peters, "Segmentation and enhancement of fingerprint images based on automatic threshold calculations," in International conference of reliable information and communication technology. Springer, 2017, pp. 400411.

[97] R. Vinayakumar, M. Alazab, K. Soman, P. Poornachandran, A. AlNemrat, and S. Venkatraman, "Deep learning approach for intelligent intrusion detection system," IEEE Access, vol. 7, pp. 41 525-41 550, 2019 .

[98] M. E. Leinonen, M. Sonkki, O. Kursu, and A. Pärssinen, "5g mmw receiver interoperability with wi-fi and lte transmissions," in 2019 13th European Conference on Antennas and Propagation (EuCAP). IEEE, 2019, pp. 1-5.

[99] P. Wang, B. Di, H. Zhang, X. Hou, and L. Song, "Cellular v2x communications in unlicensed spectrum for $5 \mathrm{~g}$ networks," in 2018
IEEE International Conference on Communications (ICC). IEEE, 2018, pp. 1-6.

[100] H. Fatemidokht, M. K. Rafsanjani, B. B. Gupta, and C.-H. Hsu, "Efficient and secure routing protocol based on artificial intelligence algorithms with uav-assisted for vehicular ad hoc networks in intelligent transportation systems," IEEE Transactions on Intelligent Transportation Systems, 2021.

[101] S. Kim, E. Visotsky, P. Moorut, K. Bechta, A. Ghosh, and C. Dietrich, "Coexistence of $5 \mathrm{~g}$ with the incumbents in the 28 and $70 \mathrm{ghz}$ bands," IEEE Journal on selected areas in communications, vol. 35, no. 6, pp. 1254-1268, 2017.

[102] F. Tian, Y. Yu, X. Yuan, B. Lyu, and G. Gui, "Predicted decoupling for coexistence between wifi and lte in unlicensed band," IEEE Transactions on Vehicular Technology, vol. 69, no. 4, pp. 4130-4141, 2020.

[103] V. Maglogiannis, A. Shahid, D. Naudts, E. De Poorter, and I. Moerman, "Enhancing the coexistence of lte and wi-fi in unlicensed spectrum through convolutional neural networks," IEEE Access, vol. 7, pp. 28 464-28 477, 2019.

[104] M. Zaeri-Amirani, F. Afghah, and S. Zeadally, "A hierarchical spectrum access scheme for tv white space coexistence in heterogeneous networks," IEEE Access, vol. 6, pp. 78 992-79 004, 2018.

[105] O. Karatalay, S. Erküçük, and T. Baykaş, "Busy tone based coexistence algorithm for wran and wlan systems in tv white space," IET Communications, vol. 12, no. 13, pp. 1630-1637, 2018.

[106] Z. Yin, Z. Li, S. M. Kim, and T. He, "Explicit channel coordination via cross-technology communication," in Proceedings of the 16th Annual International Conference on Mobile Systems, Applications, and Services, 2018, pp. 178-190.

[107] T. Xu, M. Zhang, Y. Zeng, and H. Hu, "Harmonious coexistence of heterogeneous wireless networks in unlicensed bands: solutions from the statistical signal transmission technique," IEEE Vehicular Technology Magazine, vol. 14, no. 2, pp. 61-69, 2019.

[108] R. B. Pendor and P. Tasgaonkar, "An iot framework for intelligent vehicle monitoring system," in 2016 International Conference on Communication and Signal Processing (ICCSP). IEEE, 2016, pp. 1694-1696.

[109] S. V. Bhate, P. V. Kulkarni, S. D. Lagad, M. D. Shinde, and S. Patil, "Iot based intelligent traffic signal system for emergency vehicles," in 2018 second international conference on inventive communication and computational technologies (ICICCT). IEEE, 2018, pp. 788-793.

[110] O. Avatefipour and F. Sadry, "Traffic management system using io technology-a comparative review," in 2018 IEEE International Conference on Electro/Information Technology (EIT). IEEE, 2018, pp. 1041-1047.

[111] A. Alam and Z. A. Jaffery, "Indian traffic sign detection and recognition," International Journal of Intelligent Transportation Systems Research, vol. 18, no. 1, pp. 98-112, 2020.

[112] B. K. Gandhi and M. K. Rao, "A prototype for iot based car parking management system for smart cities," Indian Journal of Science and Technology, vol. 9, no. 17, pp. 1-6, 2016.

[113] D. Elliott, W. Keen, and L. Miao, "Recent advances in connected and automated vehicles," Journal of Traffic and Transportation Engineering (English Edition), vol. 6, no. 2, pp. 109-131, 2019.

[114] M. R. T. Hossai, M. A. Shahjalal, and N. F. Nuri, "Design of an io based autonomous vehicle with the aid of computer vision," in 2017 International Conference on Electrical, Computer and Communication Engineering (ECCE). IEEE, 2017, pp. 752-756.

[115] W. Lobato, D. Rosário, M. Gerla, and L. A. Villas, "Platoon-based driving protocol based on game theory for multimedia transmission over vanet," in GLOBECOM 2017-2017 IEEE Global Communications Conference. IEEE, 2017, pp. 1-6.

[116] M. Amoozadeh, H. Deng, C.-N. Chuah, H. M. Zhang, and D. Ghosal, "Platoon management with cooperative adaptive cruise control enabled by vanet," Vehicular communications, vol. 2, no. 2, pp. 110-123, 2015.

[117] I. Grgurevic, D. Perakovic, I. Forenbacher, and T. Milinović, "Application of the internet of things concept in carsharing system," DAAAM International Scientific Book, 2015.

[118] S. Husnjak, D. Peraković, I. Forenbacher, and M. Mumdziev, "Telematics system in usage based motor insurance," Procedia Engineering, vol. 100, pp. 816-825, 2015.

[119] W. H. Organization, Guideline daily iron supplementation in infants and children. World Health Organization, 2016

[120] S. Kaivonen and E. C.-H. Ngai, "Real-time air pollution monitoring with sensors on city bus," Digital Communications and Networks, vol. 6 , no. 1, pp. 23-30, 2020. 
[121] A. Zekri and W. Jia, "Heterogeneous vehicular communications: A comprehensive study," Ad Hoc Networks, vol. 75, pp. 52-79, 2018.

[122] E. Nasr, E. Kfoury, and D. Khoury, "An iot approach to vehicle accident detection, reporting, and navigation," in 2016 IEEE International Multidisciplinary Conference on Engineering Technology (IMCET). IEEE, 2016, pp. 231-236.

[123] G. Pan, M. Muresan, R. Yu, and L. Fu, "Real-time winter road surface condition monitoring using an improved residual cnn," Canadian Journal of Civil Engineering, vol. 48, no. 9, pp. 1215-1222, 2021.

[124] B. Yao and T. Feng, "Machine learning in automotive industry," 2018.

[125] M. A. Rahman, A. T. Asyhari, I. F. Kurniawan, M. J. Ali, M. Rahman, and M. Karim, "A scalable hybrid mac strategy for traffic-differentiated iot-enabled intra-vehicular networks," Computer Communications, vol. 157, pp. $320-328,2020$

[126] M. M. Fadoul, "Rate and coverage analysis in multi-tier heterogeneous network using stochastic geometry approach," Ad Hoc Networks, vol. 98, p. 102038, 2020.

[127] A. A. Akinyelu, "Hybrid machine learning-based intelligent technique for improved big data analytics," in 2019 6th International Conference on Soft Computing \& Machine Intelligence (ISCMI). IEEE, 2019, pp. $7-11$.

[128] A. Pashazadeh and N. J. Navimipour, "Big data handling mechanisms in the healthcare applications: A comprehensive and systematic literature review," Journal of biomedical informatics, vol. 82, pp. 47-62, 2018

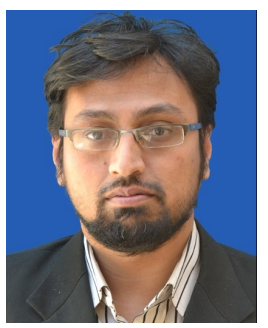

Md. Arafatur Rahman received his Ph.D. degree in Electronic and Telecommunications Engineering from the University of Naples Federico II, Naples, Italy in 2013. Currently, he is a Senior Lecturer with the School of Mathematics and Computer Science, University of Wolverhampton, UK. He was an Associate Professor with the Faculty of Computing, Universiti Malaysia Pahang, where he had conducted Undergraduate and Masters Courses and supervised more than 21 B.Sc., 5 M.Sc. and $5 \mathrm{PhD}$ students. His research interests include Internet-of-Things (IoT),

Wireless Communication Networks, Cognitive Radio Network, 5G, Vehicular Communication, Cyber-Physical System, Big Data, Cloud-Fog-Edge Computing, Machine Learning and Security.

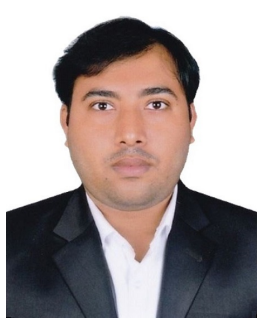

Md. Abdur Rahim received his Bachelor of Science in Mechanical Engineering from Khulna University of Engineering \& Technology (KUET), Khulna9203, Bangladesh in 2013. Currently, he is pursuing Master of Science in Automotive Engineering at Universiti Malaysia Pahang, Malaysia. His research interests are in Artificial Intelligence, Traditional, Connected, Electric and Autonomous Vehicles, Intelligent Transportation Systems, AI-based Vehicles Fault Diagnosis, Internet of Vehicles, Intelligent Robotic Systems, Big-Data Analytics, Machine Learning, Deep Learning, Renewable Energy, Energy Conservation Systems and Internal Combustion Engine.

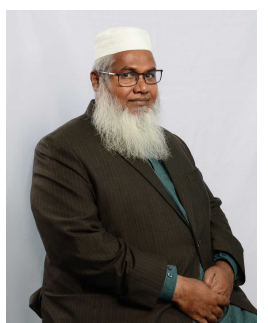

Professor Dr. Md. Mustafizur Rahman is currently working with the College of Engineering, Universiti Malaysia Pahang, Malaysia, since April 2007. Prof. Rahman also served as a Dean of Research, Research and Innovation Department, Universiti Malaysia Pahang. He received his Ph.D. degree from the Department of Mechanical and Materials Engineering, Universiti Kebangsaan Malaysia, Malaysia. The research work of Prof. Rahman is focused on Artificial Intelligence in Mechanical Systems, Applied Mechanics (Fatigue and Fracture), Computational Mechanics, Advanced Machining, Optimization, Finite Element Analysis, Modeling of Modern Materials, Internal Combustion Engine and Alternative Fuels."

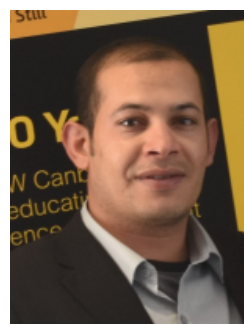

Nour Moustafa (Senior Member, IEEE) received the B.S. and M.S. degrees in computer science from the Faculty of Computer and Information, Helwan University, Egypt, in 2009 and 2014, respectively, and the Ph.D. degree in cyber security from the University of New South Wales (UNSW), Canberra in 2017. His areas of interest include cyber security, particularly network security, host- and network intrusion detection systems, statistics, deep learning, and machine learning techniques. He is currently a Lecturer in cyber security and the Coordinator of the Postgraduate Cyber Program at the School of Engineering and Information Technology (SEIT), UNSW Canberra.

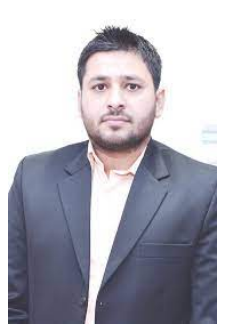

Imran Razzak (Senior Member, IEEE) has been a Senior Lecturer of computer science with the School of Information Technology, Deakin University, since November 2019. He has received numerous research awards and actively collaborating with several national and international institutes. His area of interest includes machine learning with its application spans a broad range of topics. He has applied machine learning methods with emphasis on natural language processing and image analysis to solve real-world problems related to health, finance, and social media.

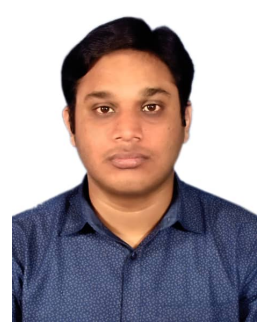

Tanvir Ahmed received his Bachelor of Science in Computer \& Communication Engineering from International Islamic University Chittagong (IIUC), Bangladesh in 2008 and Master of Science in Communication Engineering from International Islamic University Malaysia (IIUM) in 2019. Currently, he is pursuing Doctor of Philosophy (Ph.D) in System, Network and Security from University Malaysia Pahang (UMP) under the faculty of Computing. He has been serving as an IT official in Bangladesh Bank, The Central Bank of Bangladesh. His research interests are in Vehicular Networking, Intelligent Networks, Machine Learning, Cross-layer, Cyber-physical Systems, AI-based Wireless Network Security and Secured Transactions Systems.

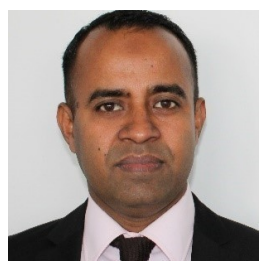

Mohammad N. Patwary (Senior Member, IEEE) received his $\mathrm{Ph} . \mathrm{D}$. in telecommunication engineering from The University of New South Wales (UNSW), Sydney, NSW, Australia, in 2005. He is currently a Professor of telecommunication networks and digital productivity with the Faculty of Science and Engineering, University of Wolverhampton, Wolverhampton, U.K. His current research interests include sensing and processing for intelligent systems, wireless communication systems design and optimization, signal processing and energy-efficient systems, future generation of cellular network architecture, and business modelling for the data economy. 